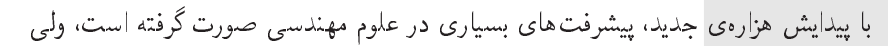

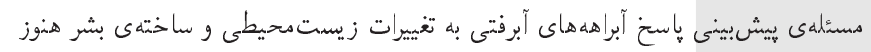

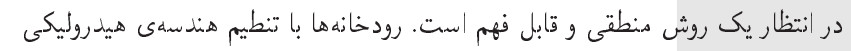

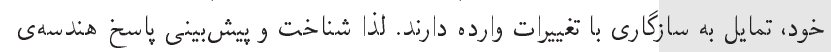

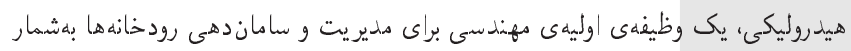

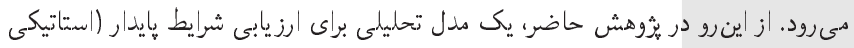

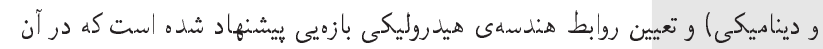

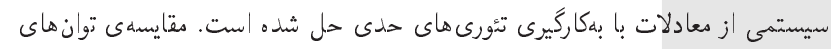

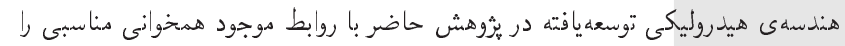

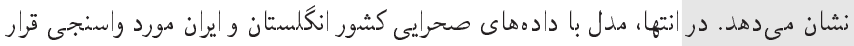

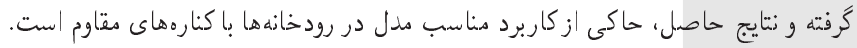

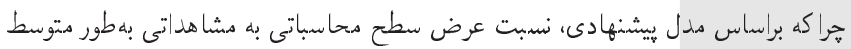

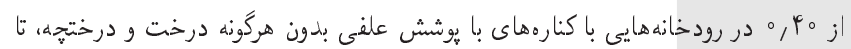
VYr

وازكان كليدى: هندسهى هيدروليكى، مدل تحليلى، تئورىهاى حدى.

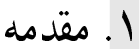

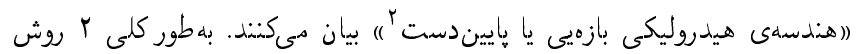

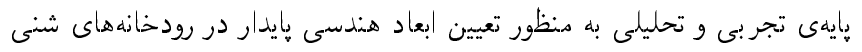

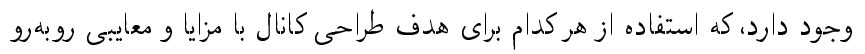

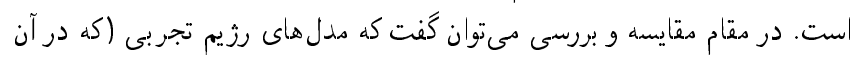

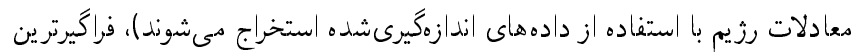

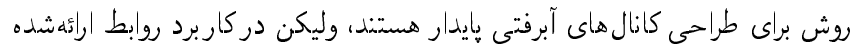

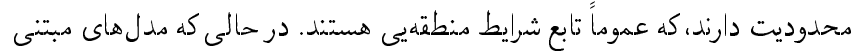

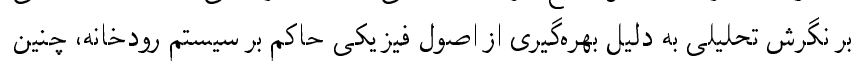

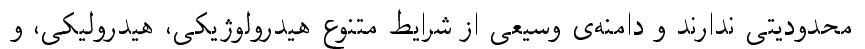

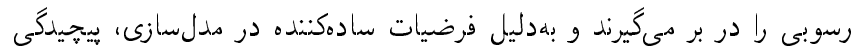

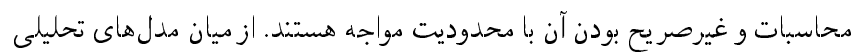

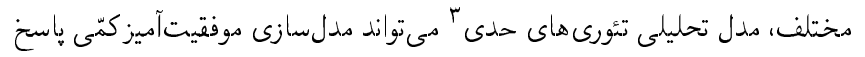

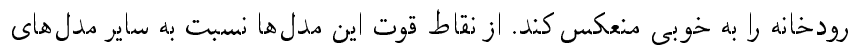

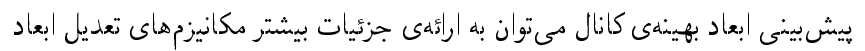

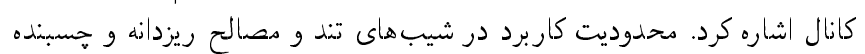

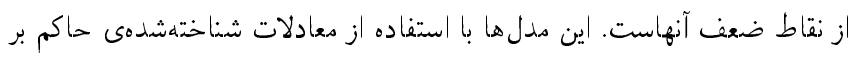

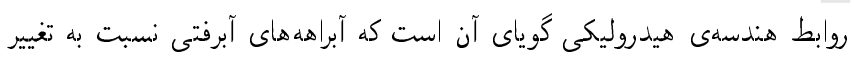

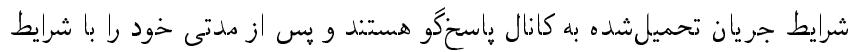

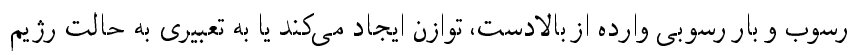

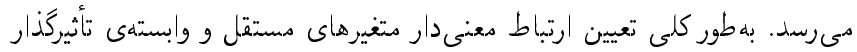

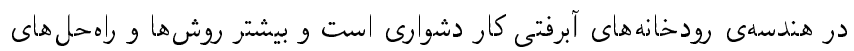

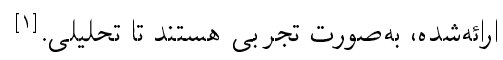

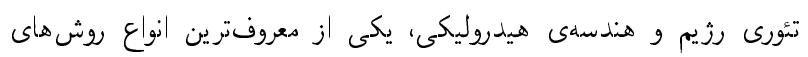

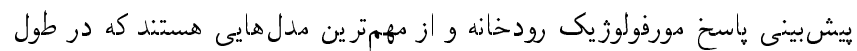

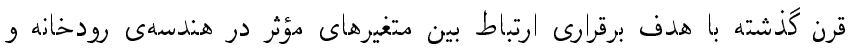

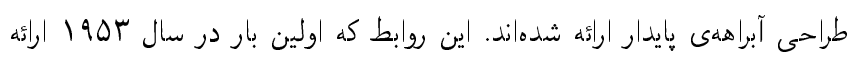

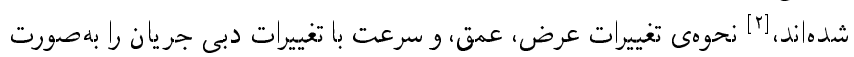

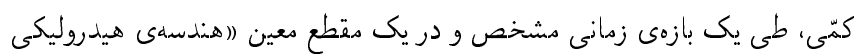

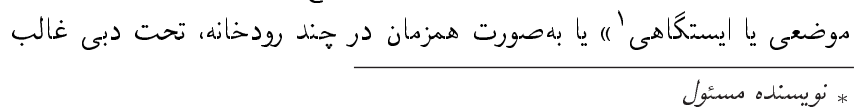
تاريخ: دريافت نو

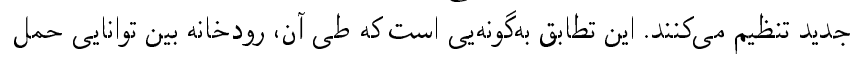


آن هستند كه دبى، دهمترين متغير مستقل كنترلكنتدهى مورفولوزى كانال است. اها در

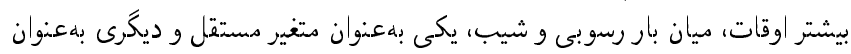

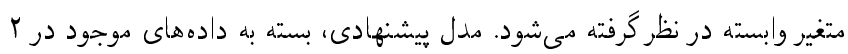

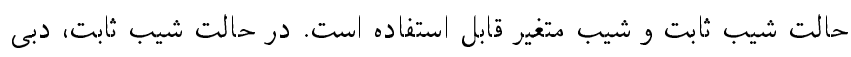

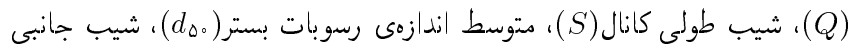

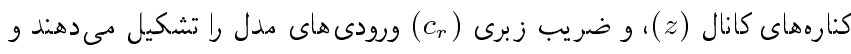

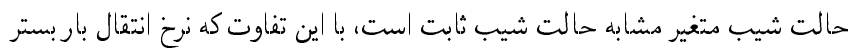
به $\left.Q_{s}\right)$

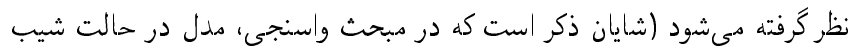

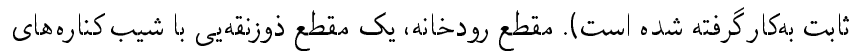

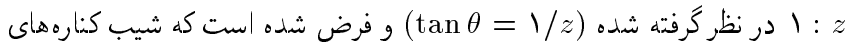

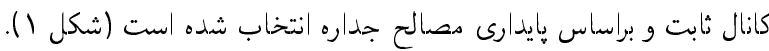

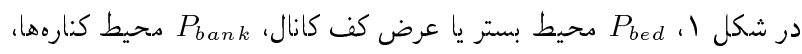

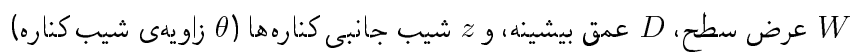

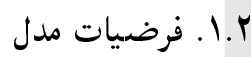
با توجه به اينكه مدلسازى رودخانههاى آبرفتى، كه سيستمهايى كاهلاً يويا هستند،

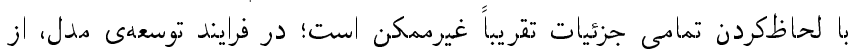
فرضيات سادهكندهيى به اين شرح استفاده شده است: 1. جريان دائمى و يكنواخت در نظر كرفته شده است؛

r. مدل ارائهشه در بازههاى تكشاخهى مستقيم، كه بار بستر در مورفولوزى آنها تأثيرگذار است، قابل كاربرد است؛

ז. دصالح آبرفتى غيرجسبنده مدنظر است؛

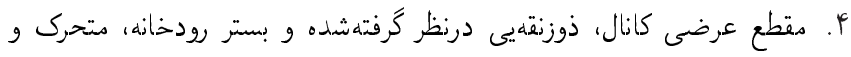

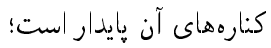

ه. شرايط بايدار و تعادلى، كه متناظر با ارضاء تئورىهاى حدى هستند، مدنظر

است؛

9. دقاوهت جريان فقط به زبرى ذرات وابسته است.

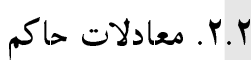

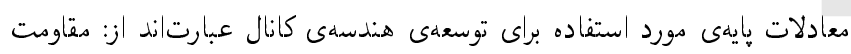

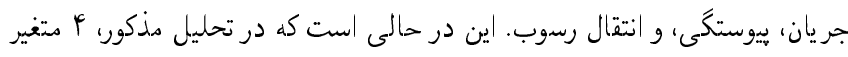

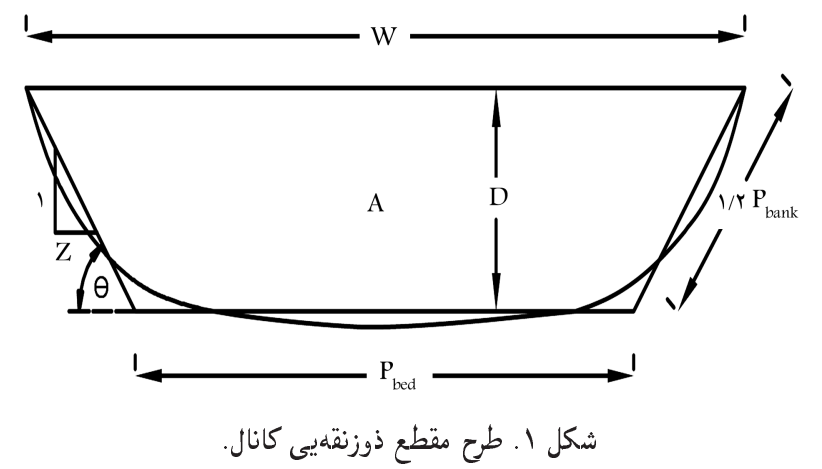

جريان نظير روابط انتقال رسوب، دقاومت جريان، و ييوستكى از يكسو و دعادلات

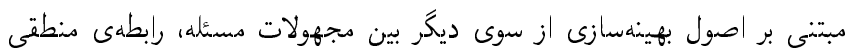

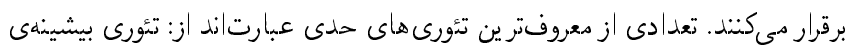

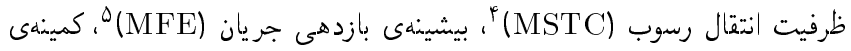
توان جريان (MSP)

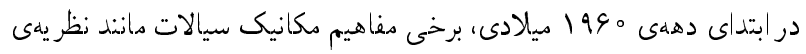

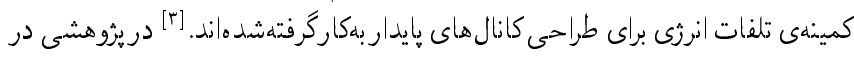

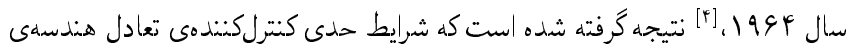

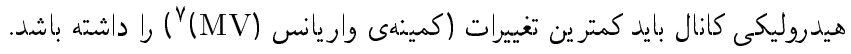

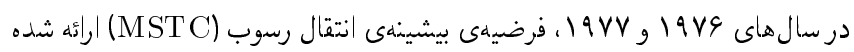

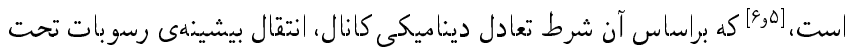

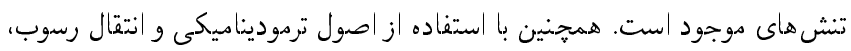

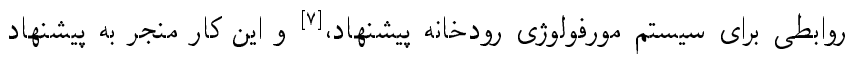

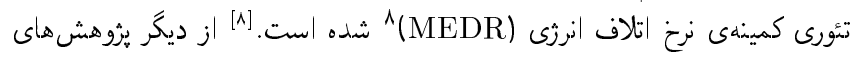

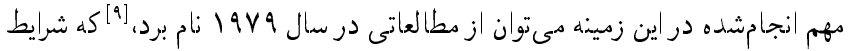

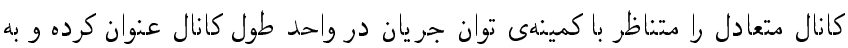

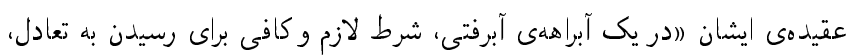

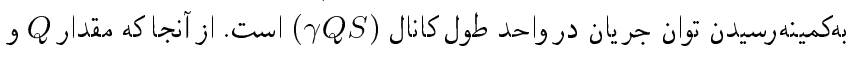

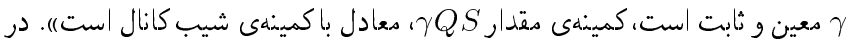

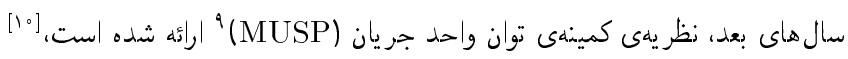

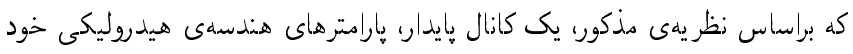

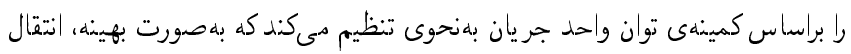

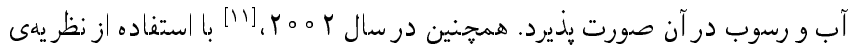

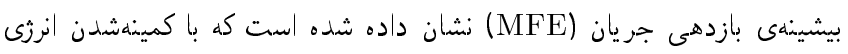

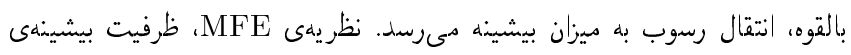

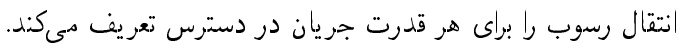

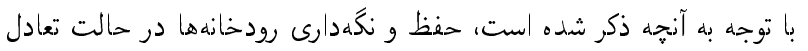

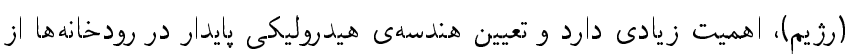

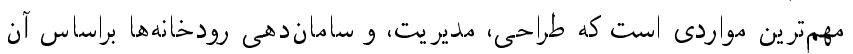

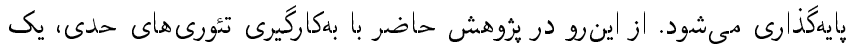

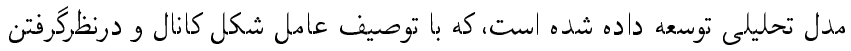
طيف وسيعى از معادلات انتقال بار بستر به فرم

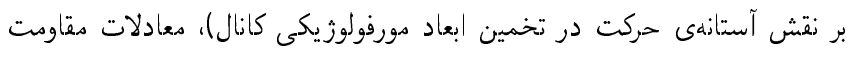

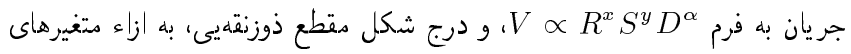

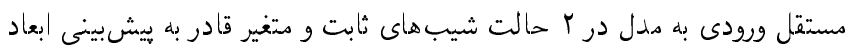

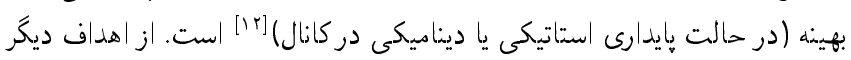

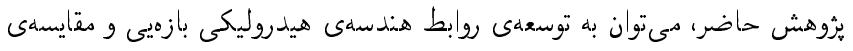

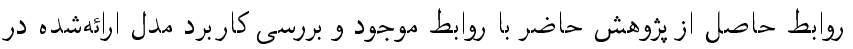
رودخانهاى طبيعى اشاره كرد.

\section{r. تئورى}

انتخاب متغيرهاى مستقل ووابسته از مسائل مهمى است كه در اغلب مدل هاى تحليلى

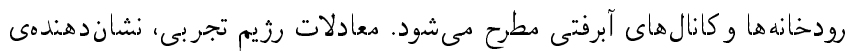




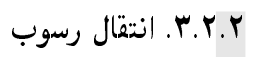

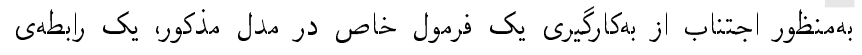

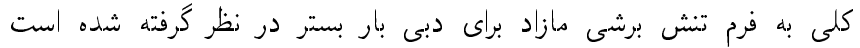

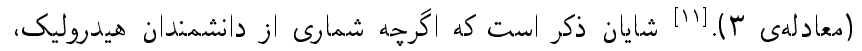

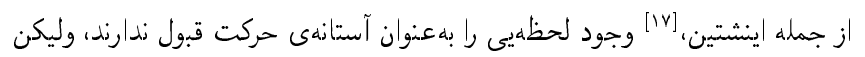

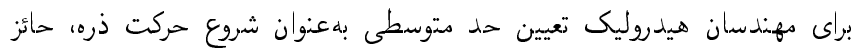

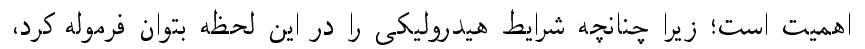

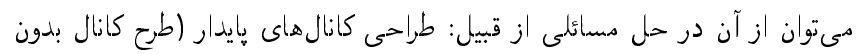

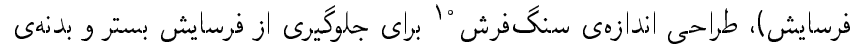

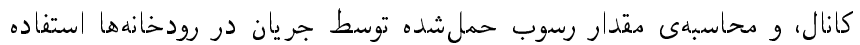
كرد:

$q_{s}=c_{s} \tau_{0}^{m}\left(\tau_{o}-\tau_{c}\right)^{j}$

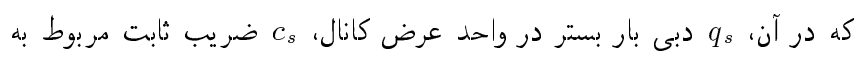

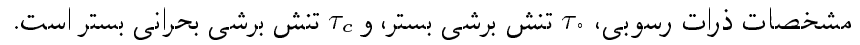

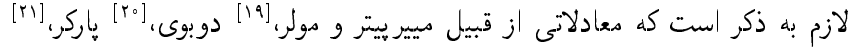

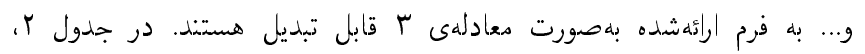

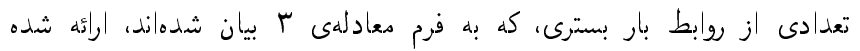

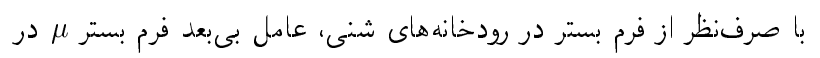

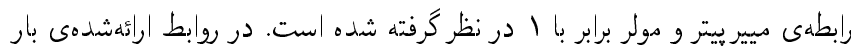

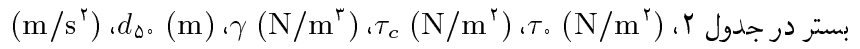

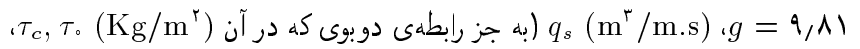

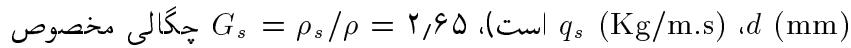

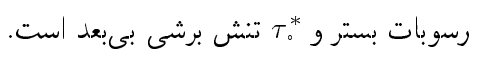

ا

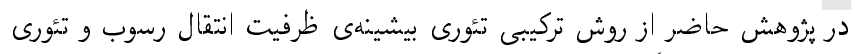

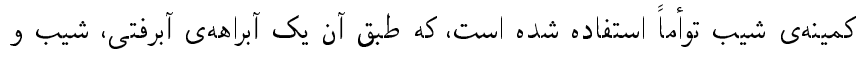

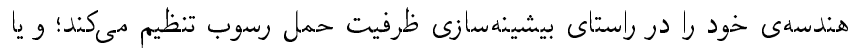

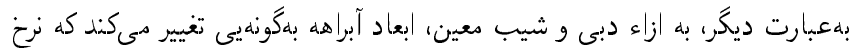

انتقال رسوب بيشينه شود.

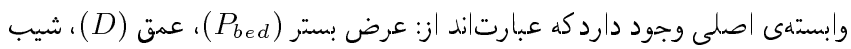

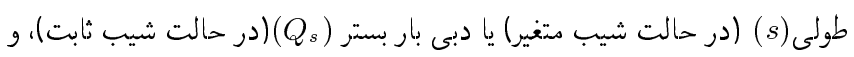

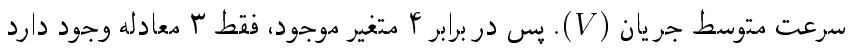

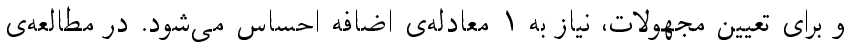

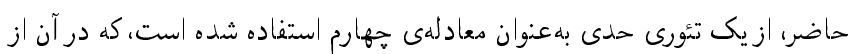
روش تركيبى بيشينهى ظرفيت انتقال رسوب وكمينهى توان جرئ إنسان (كمينهى شيب)

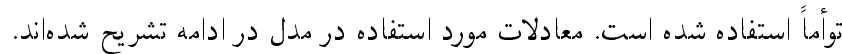

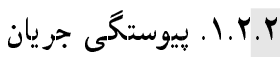
رابطهى بيوستكى براى شرايط بايدار بهصورت رابطهى إلب ارائه شده است:

$Q=A \times V$

كه در آن، Q دبى جريان غالب، V سرعت متوسط جريان، و A مساحت مقطع

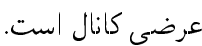

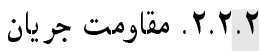

در مدل تحليلى ارائهده، فرم كلى رابطهى Y ب براى رابطهى مقاومت جريان در نظر

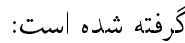

$V=c_{r} R^{x} S^{y} D^{\alpha}$

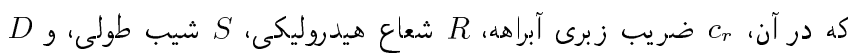

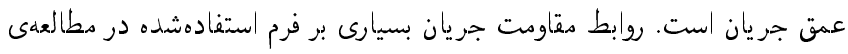

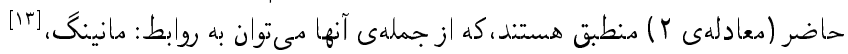

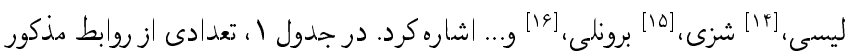

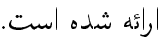

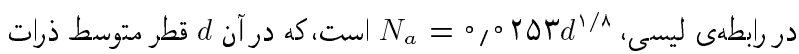
رسوبى برحسب ميلىمتر است. جدول الـ روابط مقاومت جريان.

\begin{tabular}{|c|c|c|}
\hline توانهاى $x, y, \alpha$, & رابطهى مقاومت جريان & مرجع \\
\hline$x=\frac{r}{r} ; y=\frac{1}{r} ; \alpha=0$ & $V=(1 / n) R^{r / r} S^{1 / r}$ & مانينگ [ז'] \\
\hline$x=\frac{1}{\bar{r}} ; y=\frac{1}{r} ; \alpha=\frac{1}{\bar{r}}$ & $V=\left(1 / N_{a}\right) \sqrt{R S} D^{1 / \leftarrow}$ & ليسى [ץ'] \\
\hline$x=\frac{1}{\bar{r}} ; y=\frac{1}{\mathrm{r}} ; \alpha=0$ & $V=c R^{1 / r} S^{1 / r}$ & شزي [10] \\
\hline
\end{tabular}

جدول r. معادلات بار بستر مورد استفاده به فرم تنش برشى مازاد.

\begin{tabular}{|c|c|c|c|}
\hline ضرايب و توانها & فرم تغييريافتهى رابطهى بار بستر & رابطهى بار بستر & 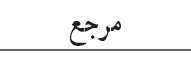 \\
\hline $\begin{array}{c}c_{s}=\wedge \frac{\sqrt{g\left(G_{s}-1\right) d_{\delta_{\diamond}}^{\natural}}}{\left(\gamma d_{{ }_{0}}\left(G_{s}-1\right)\right)^{1 / \diamond}} \\
m=0 ; j=1,0\end{array}$ & $q_{s}=c_{s}\left(\tau_{\circ}-\tau_{c}\right)^{1 / 0}$ & 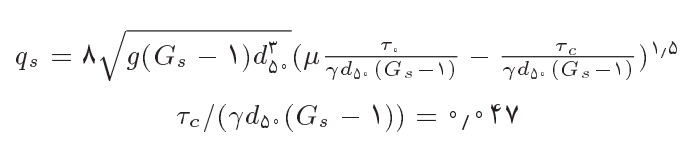 & مييرييتر و مولر [19] \\
\hline $\begin{array}{c}c_{s}=c_{d} \\
m=1 ; j=1\end{array}$ & $q_{s}=c_{s} \tau_{\circ}\left(\tau_{\circ}-\tau_{c}\right)$ & $\begin{array}{c}q_{s}=c_{d} \tau_{0}\left(\tau_{\circ}-\tau_{c}\right) \\
\tau_{c}=0,091+0,09 r_{d} ; c_{d}=0, \backslash \vee d^{-r / \digamma} ; \tau_{\circ}=\rho R S\end{array}$ & دوبوى [י[م] \\
\hline $\begin{array}{c}c_{s}=11, r \frac{\sqrt{g\left(G_{s}-1\right) d_{\sigma_{c}}^{r}}}{\left(\gamma d_{\partial_{c}}\left(G_{s}-1\right)\right)^{1 / \Delta}} \\
m=-r ; j=r, \Delta\end{array}$ & $q_{s}=c_{s}\left(\tau_{o}-\tau_{c}\right)^{\tau, \Delta} / \tau_{o}^{r}$ & 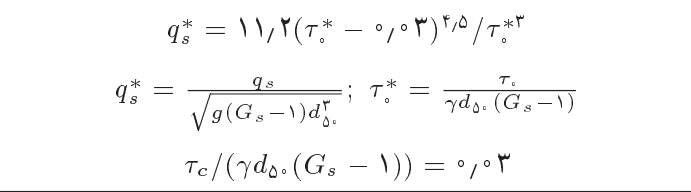 & 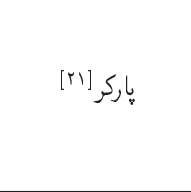 \\
\hline
\end{tabular}


با مشتقگيرى جزئى از

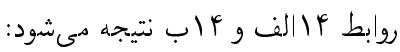

$\frac{\partial P_{b e d}}{\partial \zeta}=\left[\frac{(1+x+\alpha) \zeta+r(1+\alpha) \sqrt{1+z^{r}}}{\left(\zeta+r \sqrt{1+z^{r}}\right)(\zeta+z)(r+x+\alpha)}\right.$

$\left.+\frac{(r+r x+\alpha) z+\frac{(\digamma+r x+r \alpha) z \sqrt{1+z^{\Upsilon}}}{\zeta}}{\left(\zeta+r \sqrt{1+z^{r}}\right)(\zeta+z)(r+x+\alpha)}\right] P_{b e d}$

(الف)

$\frac{\partial P_{b e d}}{\partial S}=\frac{-y}{(\Upsilon+x+\alpha) S} P_{b e d}$

$\frac{\partial \tau_{\circ}}{\partial \zeta}=\frac{-\zeta+r(1+\alpha) \sqrt{1+z^{r}}-(r+\alpha) z}{\left(\zeta+r \sqrt{1+z^{r}}\right)(\zeta+z)(r+x+\alpha)} \tau_{0}$

$\frac{\partial \tau_{\circ}}{\partial S}=\frac{(r+x+\alpha-y)}{(r+x+\alpha) S} \tau_{0}$

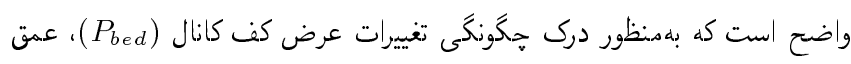

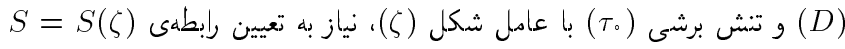

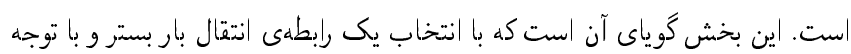

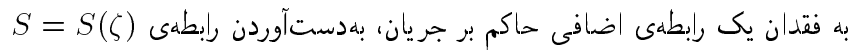

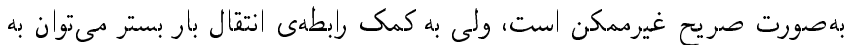
نحوهى تغييرات

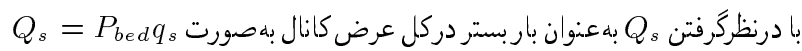

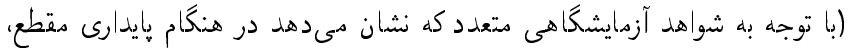

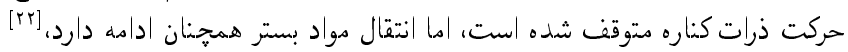

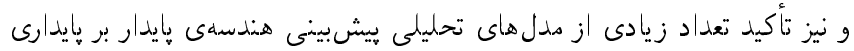

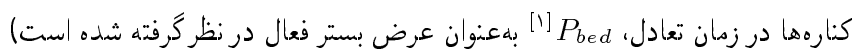
و تركيب آن با روابط (ك)

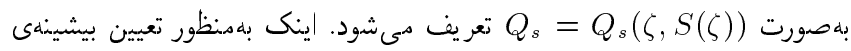

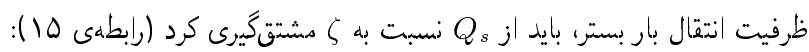

$\frac{d Q_{s}}{d \zeta}=\frac{\partial Q_{s}}{\partial \zeta}+\frac{\partial Q_{s}}{\partial S} \frac{d S}{d \zeta}$

با درنظركرفتن رابطهى

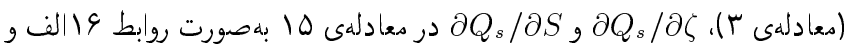

$$
\begin{aligned}
\frac{\partial Q_{s}}{\partial \zeta} & =\frac{\partial Q_{s}}{\partial \tau_{\circ}} \frac{\partial \tau_{\circ}}{\partial \zeta}+\frac{\partial Q_{s}}{\partial P_{b e d}} \frac{\partial P_{b e d}}{\partial \zeta} \\
& =Q_{s}\left[\left(\frac{m}{\tau_{\circ}}+\frac{j}{\left(\tau_{\circ}-\tau_{c}\right)}\right) \frac{\partial \tau_{\circ}}{\partial \zeta}+\frac{1}{P_{b e d}} \frac{\partial P_{b e d}}{\partial \zeta}\right] \\
\frac{\partial Q_{s}}{\partial S} & =\frac{\partial Q_{s}}{\partial \tau_{\circ}} \frac{\partial \tau_{\circ}}{\partial S}+\frac{\partial Q_{s}}{\partial P_{b e d}} \frac{\partial P_{b e d}}{\partial S} \\
& =Q_{s}\left[\left(\frac{m}{\tau_{\circ}}+\frac{j}{\left(\tau_{\circ}-\tau_{c}\right)}\right) \frac{\partial \tau_{\circ}}{\partial S}+\frac{1}{P_{b e d}} \frac{\partial P_{b e d}}{\partial S}\right]
\end{aligned}
$$

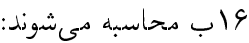

در ادامه، با جايكذارى عبارات

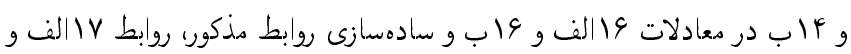

\section{r.r.r. توسعهى مدل تحليلى}

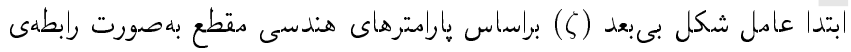
F أتعريف شده است:

$\zeta=P_{b e d} / D$

كه در آن،

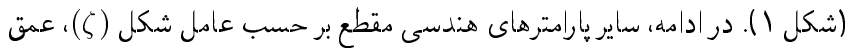

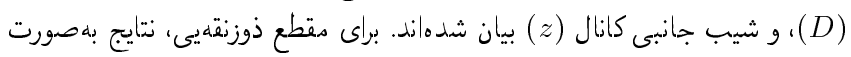
روابط ه الى ^ ه است:

$A=D^{r}(\zeta+z)$

$P=P_{b e d}+P_{b a n k}=D\left(\zeta+r \sqrt{1+z^{r}}\right)$

$R=A / P=\frac{(\zeta+z)}{\left(\zeta+r \sqrt{1+z^{r}}\right)} D$

$W=P_{b e d}+r z D=D(\zeta+r z)$

كه در آنها، A، P، R و W W به ترتيب مساحت مقطع عرضى، دحيط ترشدهى

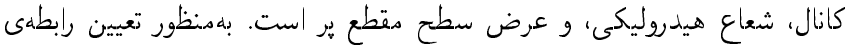

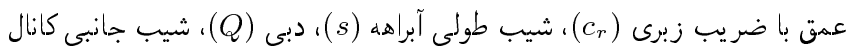

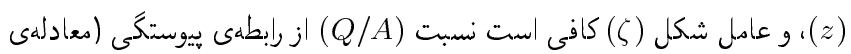

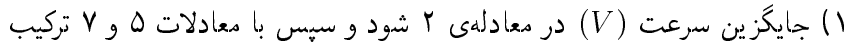

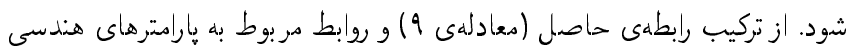

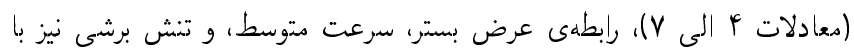

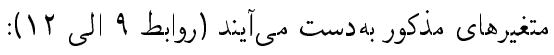

$D=\frac{\left(\zeta+r \sqrt{1+z^{r}}\right)^{x /(x+r+\alpha)}}{(\zeta+z)^{(x+1) /(x+r+\alpha)}} \frac{\left(Q / c_{r}\right)^{1 /(x+r+\alpha)}}{S^{y /(x+r+\alpha)}}$

$P_{b e d}=(\zeta D)=\frac{\zeta\left(\zeta+r \sqrt{1+z^{r}}\right)^{x /(x+r+\alpha)}}{(\zeta+z)^{(x+1) /(x+r+\alpha)}} \frac{\left(Q / c_{r}\right)^{1 /(x+r+\alpha)}}{S^{y /(x+r+\alpha)}}$

$V=(Q / A)=\frac{\left(S^{r y} Q^{(x+\alpha)} c_{r}^{r}\right)^{1 /(x+r+\alpha)}(\zeta+z)^{(x-\alpha /(x+r+\alpha)}}{\left(\zeta+r \sqrt{1+z^{r}}\right)^{(r x) /(x+r+\alpha)}}$

$\tau_{\circ}=(\gamma R S)$

$$
=\frac{\gamma S^{1-(y /(x+r+\alpha))}\left(\frac{Q}{c_{r}}\right)^{1 /(x+r+\alpha)}(\zeta+z)^{(1+\alpha) /(x+r+\alpha)}}{\left(\zeta+r \sqrt{1+z^{r}}\right)^{(r+\alpha) /(x+r+\alpha)}}
$$

در اين قسمت از مدلسازى، در ابتدا شيب طولى كانال بهصورت تابعى از عامل شكل كانال (S) r T。 $=\tau_{\circ}(\zeta, S(\zeta))$ داشت:

$$
\frac{d P_{b e d}}{d \zeta}=\frac{\partial P_{b e d}}{\partial \zeta}+\frac{\partial P_{b e d}}{\partial S} \frac{d S}{d \zeta}
$$

$\frac{d \tau_{\circ}}{d \zeta}=\frac{\partial \tau_{\circ}}{\partial \zeta}+\frac{\partial \tau_{\circ}}{\partial S} \frac{d S}{d \zeta}$ 


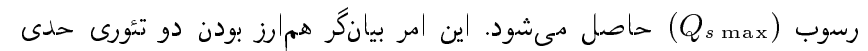

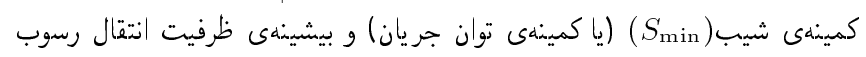

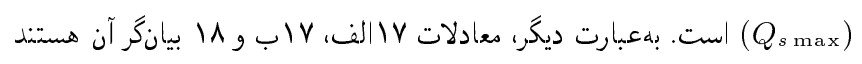

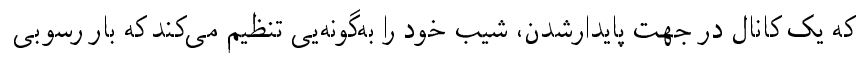

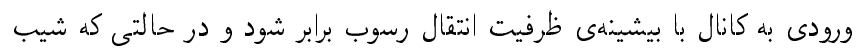

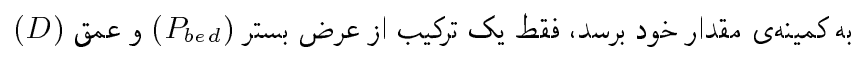

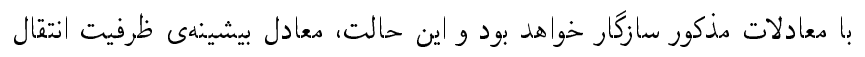

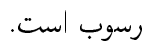

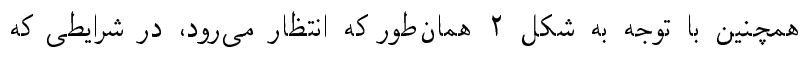

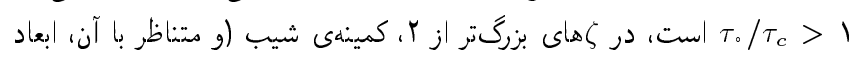
بهينهى كانال) حاصل مىشود (r) (

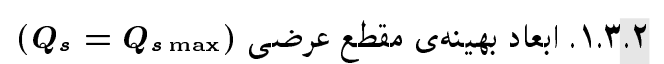

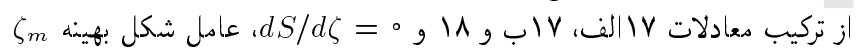

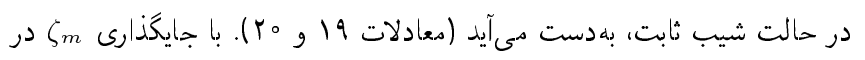

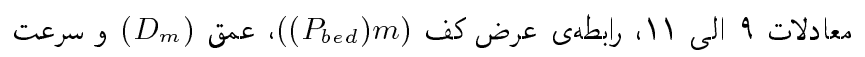

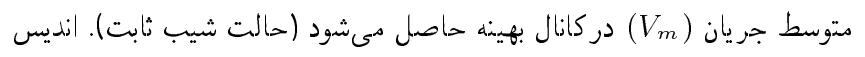
m m، نشاندهندمى شرايط بهينه در كانال است.

$$
\begin{aligned}
& \frac{\tau_{\circ}}{\tau_{c}}=\frac{K_{\mathrm{r}}}{K_{\backslash}} \\
& \frac{\tau_{\circ}-\tau_{c}}{\tau_{c}}=\frac{j\left(\zeta_{m}+(\boldsymbol{\Gamma}+\alpha) z-\Upsilon(1+\alpha) \sqrt{1+z^{\mathrm{r}}}\right)}{K_{\backslash}}
\end{aligned}
$$

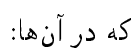

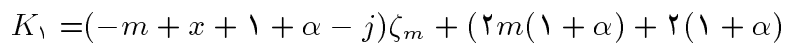

$$
\begin{aligned}
& +\boldsymbol{r} j(\boldsymbol{I}+\alpha)) \sqrt{1+z^{\boldsymbol{r}}}+(-m(\boldsymbol{r}+\alpha) \boldsymbol{r}+\boldsymbol{r} x+\alpha \\
& -j(\boldsymbol{r}+\alpha)) z+\frac{(\boldsymbol{\Psi}+\boldsymbol{r} x+\boldsymbol{r} \alpha) z \sqrt{\boldsymbol{1 + z ^ { \boldsymbol { r } }}}}{\zeta_{m}}
\end{aligned}
$$

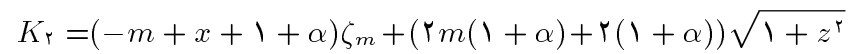

$$
+(-m(\boldsymbol{r}+\alpha)+\boldsymbol{r}+\boldsymbol{r} x+\alpha) z+\frac{(\boldsymbol{\Psi}+\boldsymbol{r} x+\boldsymbol{r} \alpha) z \sqrt{\boldsymbol{1 + z ^ { \boldsymbol { r } }}}}{\zeta_{m}}
$$

لازم به ذكر است كه در ادامه، ضرايب >K و rK از معادلهى 19 قابل محاسبه

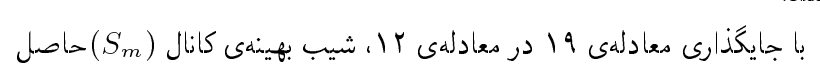

$$
\text { مىشود (رابطهى (r) }
$$

$$
\begin{aligned}
& S_{m}=\left(\left(c_{r} / Q\right)\left(\tau_{c} / \gamma\right)^{(x+r+\alpha)}\right)^{1 /(x+r+\alpha-y)} \\
& \times\left(\frac{K_{r}}{K_{1}}\right)^{\frac{(x+r+\alpha)}{(x+r+\alpha-y)}} \frac{\left(\zeta_{m}+r \sqrt{1+z^{r}}\right)^{(r+\alpha) /(x+r+\alpha-y)}}{\left(\zeta_{m}+z\right)^{(1+\alpha) /(x+r+\alpha-y)}}
\end{aligned}
$$

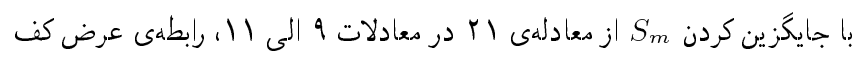
(

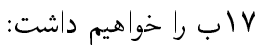

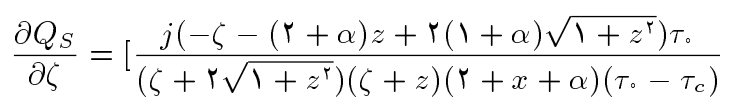

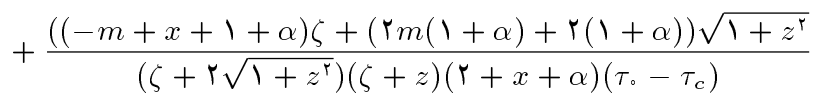

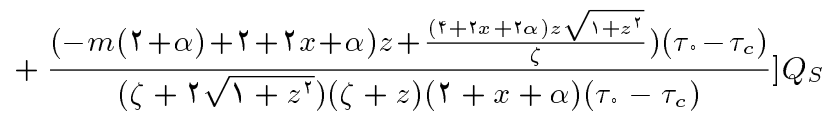

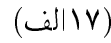

$\frac{\partial Q_{S}}{\partial S}=$

$\frac{[(x+\boldsymbol{Y}+\alpha-y)(m+j)-y] \tau_{\circ}+[y-m(x+\boldsymbol{\Gamma}+\alpha-y)] \tau_{c}}{S(\boldsymbol{\Gamma}+x+\alpha)\left(\tau_{\circ}-\tau_{c}\right)} Q_{S}$

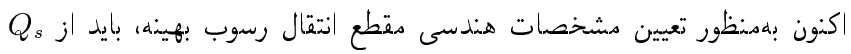

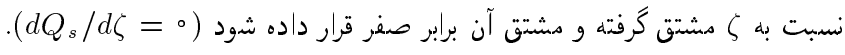

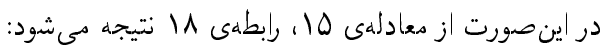

$\frac{d S}{d \zeta}=-\frac{\partial Q_{s} / \partial \zeta}{\partial Q_{s} / \partial S}$

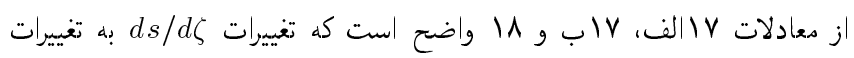
درن

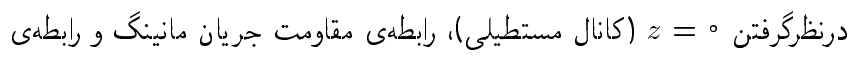

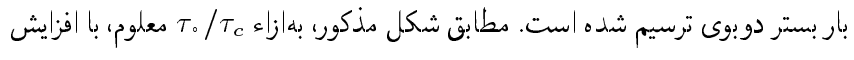

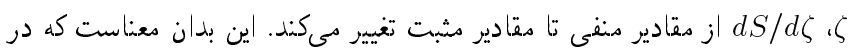

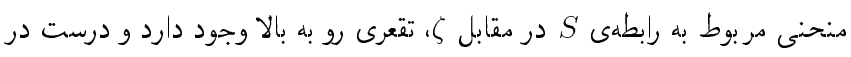

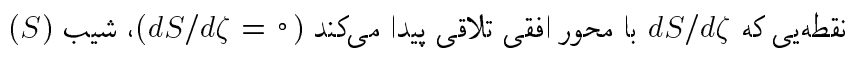

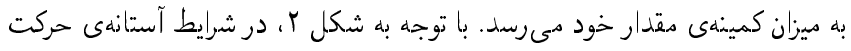

.

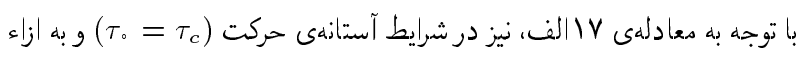

و

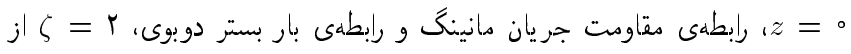

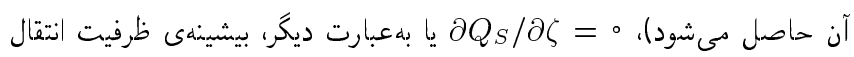

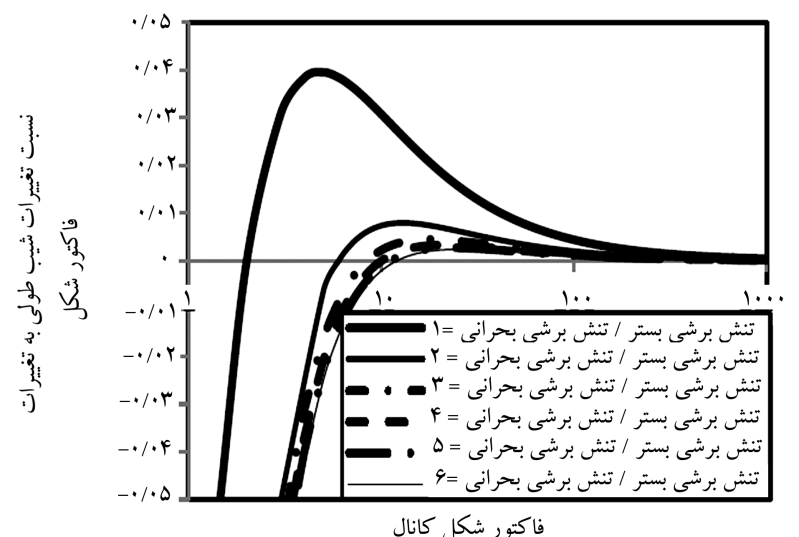

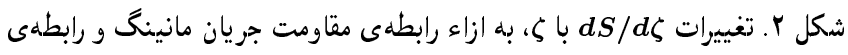

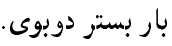


حد بايينى mك، نشاندهندهى شرايط آستانهى حركت و حد بالايى آن نشاندهندهى

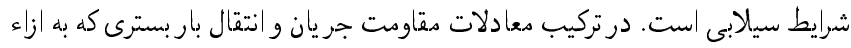

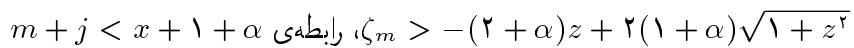

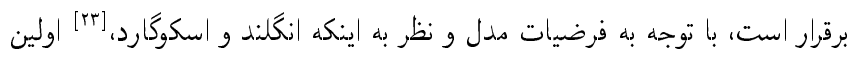

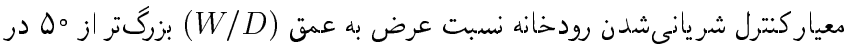
نظر كرفته شده است، حد بالايى

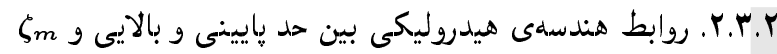

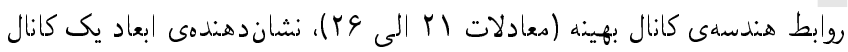

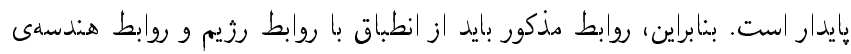

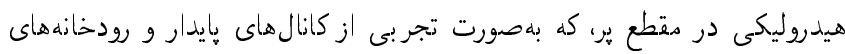

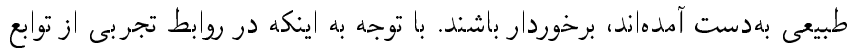

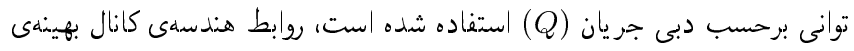

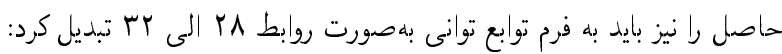

$S_{m} \propto Q^{-1 /(x+\gamma+\alpha-y)} \zeta_{m}^{\beta,}$

$\left(P_{b e d}\right)_{m} \propto Q^{\frac{1}{(x+r+\alpha-y)}} \zeta_{m}^{\beta_{\mathrm{r}}}$

$Q_{s} \propto Q^{\frac{1}{(x+\mathrm{r}+\alpha-y)} \zeta_{m}^{\beta_{\mathrm{r}}}}$

$V_{m} \propto Q^{\frac{(x+\alpha-y)}{(x+++\alpha-y)}} \zeta_{m}^{\beta_{\varphi}}$

$W_{m} \propto Q^{\frac{1}{(x+r+\alpha-y)}} \zeta_{m}^{\beta_{\triangleright}}$

كه در آنها، $\zeta_{m}^{\beta_{l}} \propto\left(\frac{K_{r}}{K_{l}}\right)^{\frac{(x+r+\alpha)}{(x+r+\alpha-y)}} \frac{\left(\zeta_{m}+r \sqrt{1+z^{r}}\right)^{(r+\alpha) /(x+r+\alpha-y)}}{\left(\zeta_{m}+z\right)^{(1+\alpha) /(x+r+\alpha-y)}}$ $\zeta_{m}^{\beta_{\Upsilon}} \propto\left(\frac{K_{\zeta}}{K_{\mathrm{r}}}\right)^{\frac{y}{(x+\mathrm{r}+\alpha-y)}} \frac{\zeta_{m}\left(\zeta_{m}+\mathrm{r} \sqrt{1+z^{\Upsilon}}\right)^{(x-y) /(x+\mathrm{r}+\alpha-y)}}{\left(\zeta_{m}+z\right)^{(x-y+1) /(x+\mathrm{r}+\alpha-y)}}$ $\zeta_{m}^{\beta_{r}} \propto \frac{\zeta_{m}\left(\zeta_{m}+r \sqrt{1+z^{r}}\right)^{(x-y) /(x+r+\alpha-y)}}{\left(\zeta_{m}+z\right)^{(x-y+1) /(x+r+\alpha-y)}}$

$$
\times \frac{\left(\zeta_{m}+(\boldsymbol{\Gamma}+\alpha) z-\boldsymbol{\Gamma}(1+\alpha) \sqrt{1+z^{\Upsilon}}\right)^{j}\left(K_{\mathrm{r}}\right)^{m-\frac{y}{(x+\boldsymbol{\Gamma}+\alpha y)}}}{\left(K_{\uparrow}\right)^{m+j-\frac{y}{(x+\mathrm{r}+\alpha-y)}}}
$$

$\zeta_{m}^{\beta_{\varphi}} \propto\left(\frac{K_{r}}{K_{l}}\right)^{\frac{r_{y}}{(x+r+\alpha-y)}} \frac{\left(\zeta_{m}+z\right)^{(x-y-\alpha) /(x+r+\alpha-y)}}{\left(\zeta_{m}+r \sqrt{1+z^{r}}\right)^{\Upsilon(x-y) /(x+r+\alpha-y)}}$ $\zeta_{m}^{\beta_{\Delta}} \propto\left(\frac{K_{\downarrow}}{K_{\mathrm{r}}}\right)^{\frac{y}{(x+r+\alpha-y)}} \frac{\left(\zeta_{m}+r \sqrt{1+z^{r}}\right)^{(x-y) /(x+r+\alpha-y)}\left(\zeta_{m}+r z\right)}{\left(\zeta_{m}+z\right)^{(x-y+1) /(x+r+\alpha-y)}}$

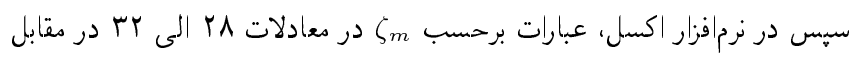

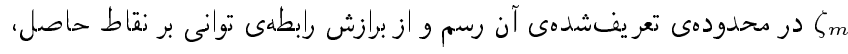

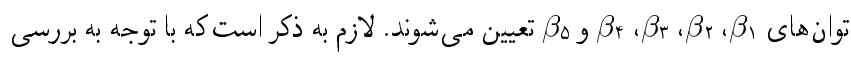

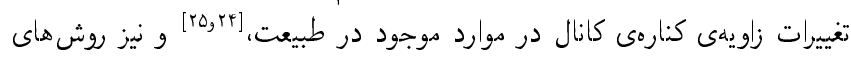

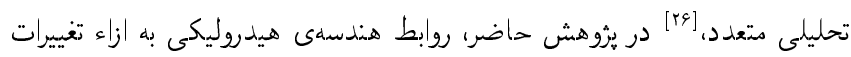

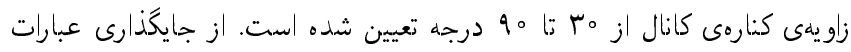

$$
\begin{aligned}
\left(P_{b e d}\right)_{m}= & \left(\frac{Q \gamma^{y}}{c_{r} \tau_{c}^{y}}\right)^{(x+\mathrm{r}+\alpha-y)} \times\left(\frac{K_{1}}{K_{r}}\right)^{(x+\mathrm{r}+\alpha-y)} \\
& \frac{\zeta_{m}\left(\zeta_{m}+\mathrm{r} \sqrt{1+z^{r}}\right)^{(x-y) /(x+r+\alpha-y)}}{\left(\zeta_{m}+z\right)^{(x-y+1) /(x+\Upsilon+\alpha-y)}}
\end{aligned}
$$

$$
\begin{aligned}
D_{m}= & \left(\frac{Q \gamma^{y}}{c_{r} \tau_{c}^{y}}\right)^{\frac{1}{(x+\mathrm{r}+\alpha-y)}} \times\left(\frac{K_{1}}{K_{\mathrm{r}}}\right)^{\frac{y}{(x+\mathrm{r}+\alpha-y)}} \\
& \frac{\left(\zeta_{m}+\mathrm{r} \sqrt{1+z^{r}}\right)^{(x-y) /(x+\mathrm{r}+\alpha-y)}}{\left(\zeta_{m}+z\right)^{(x-y+1) /(x+\mathrm{r}+\alpha-y)}}
\end{aligned}
$$

$V_{m}=\left(Q^{(x+\alpha-y)} c_{r}^{r}\left(\frac{\tau_{c}}{\gamma}\right)^{r y}\right)^{\frac{1}{(x+r+\alpha-y)}} \times\left(\frac{K_{r}}{K_{\curlyvee}}\right)^{\frac{r_{y}}{(x+r+\alpha-y)}}$

$$
\frac{\left(\zeta_{m}+z\right)^{(x-y-\alpha) /(x+\Upsilon+\alpha-y)}}{\left(\zeta_{m}+\Upsilon \sqrt{1+z^{r}}\right)^{\Upsilon(x-y) /(x+\Upsilon+\alpha-y)}}
$$

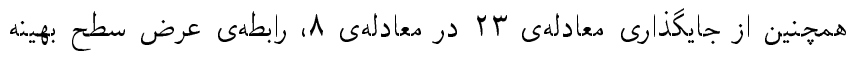

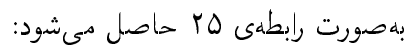

$W_{m}=\left(\frac{Q \gamma^{y}}{c_{r} \tau_{c}^{y}}\right)^{\frac{1}{(x+\mathrm{r}+\alpha-y)}}\left(\frac{K_{1}}{K_{\mathrm{r}}}\right)^{\frac{y}{(x+\mathrm{r}+\alpha-y)}}$

$$
\times \frac{\left(\zeta_{m}+r \sqrt{1+z^{r}}\right)^{(x-y) /(x+r+\alpha-y)}\left(\zeta_{m}+\Upsilon z\right)}{\left(\zeta_{m}+z\right)^{(x-y+1) /(x+r+\alpha-y)}}
$$

با درنظركرفتن رابطهى بار بستر دركل عرض كانال بهصورت:

$$
Q_{s}=P_{b e d} c_{s}\left(\tau_{\circ} / \tau_{c}\right)^{m}\left(\left(\tau_{\circ}-\tau_{c}\right) / \tau_{c}\right)^{j} \tau_{c}^{m+j}
$$

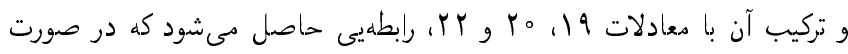

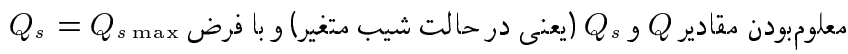
در شرايط تعادل در كانال، عامل شكل بهينهى كانال (

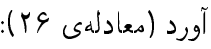

$Q_{s}=\left(Q_{s \max }\right)=j^{j} c_{s} \gamma^{(x+Y+\alpha-y)} \tau_{c}^{m+j-\frac{y}{(x+\mathrm{Y}+\alpha-y)}}\left(Q / c_{r}\right)^{\frac{1}{(x+\mathrm{Y}+\alpha-y)}}$

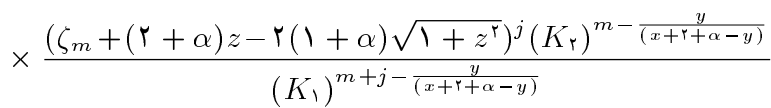

$$
\begin{aligned}
& \times \frac{\zeta_{m}\left(\zeta_{m}+r \sqrt{1+z^{r}}\right)^{(x-y) /(x+r+\alpha-y)}}{\left(\zeta_{m}+z\right)^{(x-y+1) /(x+r+\alpha-y)}}
\end{aligned}
$$

مطابق معادلهى צr به، به ازاء

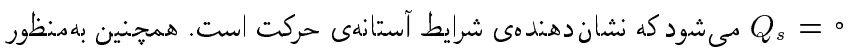
حاصلشدن مقادير 0

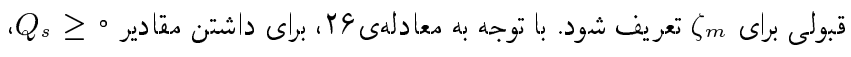

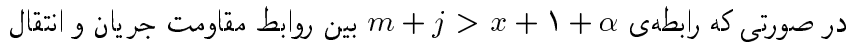

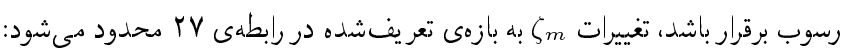

$$
\begin{aligned}
& -(\boldsymbol{Y}+\alpha) z+\Upsilon(1+\alpha) \sqrt{1+z^{r}}<\zeta_{m}< \\
& {\left[\frac{(r m(1+\alpha)+\Upsilon(1+\alpha)+r j(1+\alpha)) \sqrt{1+z^{r}}}{\Gamma(m-x-1-\alpha+j)}\right.}
\end{aligned}
$$

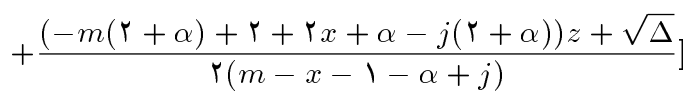




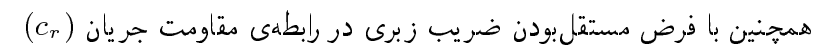

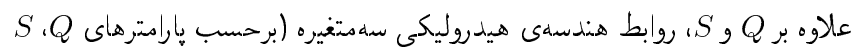

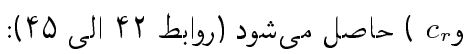

$\left(P_{b e d}\right)_{m} \propto S^{\beta_{\curlyvee} / \beta_{\uparrow}}\left(c_{r}^{-1} Q\right)^{\left(1+\left(\beta_{\curlyvee} / \beta_{\uparrow}\right)\right) /(x+r+\alpha-y)}$

$D_{m} \propto S^{\left(\beta_{\Upsilon}-1\right) / \beta_{\backslash}}\left(c_{r}^{-1} Q\right)^{\left(1+\left(\left(\beta_{\Upsilon}-1\right) / \beta_{\uparrow}\right)\right) /(x+r+\alpha-y)}$

$V_{m} \propto S^{\beta_{\digamma} / \beta_{\curlywedge}} Q^{\left(x+\alpha-y+\left(\beta_{\digamma} / \beta_{\curlywedge}\right)\right) /(x+r+\alpha-y)} c_{r}^{\left(r-\left(\beta_{\digamma} / \beta_{\uparrow}\right)\right) /(x+r+\alpha-y)}$

$W_{m} \propto S^{\beta_{\diamond} / \beta_{\curlywedge}}\left(c_{r}^{-} \mathcal{Q} Q\right)^{\left(1+\left(\beta_{\diamond} / \beta_{\curlywedge}\right)\right) /(x+r+\alpha-y)}$

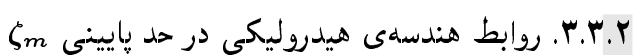
هادلهى

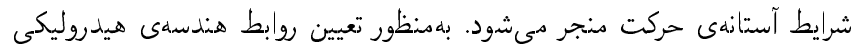

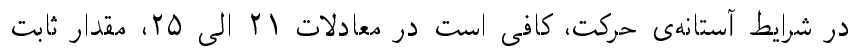

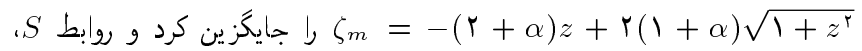
أرد، $D$

$$
\begin{aligned}
& S_{t r}=t_{t r} Q^{-1 /(x+r+\alpha-y)} \\
& D_{t r}=c_{t r} Q^{1 /(x+r+\alpha-y)} \\
& V_{t r}=k_{t r} Q^{(x+\alpha-y) /(x+r+\alpha-y)} \\
& W_{t r}=a_{t r} Q^{1 /(x+r+\alpha-y)}
\end{aligned}
$$$$
\text { آورد: }
$$

كه در آن ها، ضرايب a

$$
\begin{aligned}
& a_{t r}=\left(\gamma^{y} /\left(c_{r} \tau_{c}^{y}\right)\right)^{1 /(x+\Upsilon+\alpha-y)}\left(\Upsilon(1+\alpha) \sqrt{1+z^{r}}-\alpha z\right) \\
& \times \frac{\left(\boldsymbol{r}(\boldsymbol{I}+\alpha) \sqrt{1+z^{\Upsilon}}-z(\boldsymbol{I}+\alpha)\right)^{(y-x-1) /(x+\boldsymbol{r}+\alpha-y)}}{\left((\boldsymbol{\Upsilon}+\boldsymbol{r} \alpha) \sqrt{1+z^{r}}-z(\boldsymbol{r}+\alpha)\right)^{(y-x) /(x+\boldsymbol{r}+\alpha-y)}} \\
& c_{t r}=\left(\frac{\gamma^{y}}{c_{r} \tau_{c}^{y}}\right)^{\frac{1}{(x+\gamma+\alpha-y)}}
\end{aligned}
$$

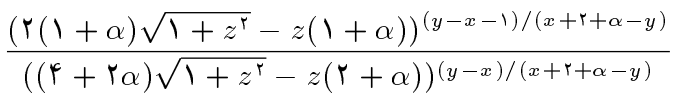

$$
k_{t r}=\left(c_{r}^{r}\left(\tau_{c} / \gamma\right)^{r y}\right)^{\frac{1}{(x+r+\alpha-y)}}
$$

$$
\frac{\left(\Upsilon(1+\alpha) \sqrt{1+z^{\Upsilon}}-(\backslash+\alpha) z\right)^{\frac{(-y+x-\alpha)}{(x+\Upsilon+\alpha-y)}}}{\left((\boldsymbol{\Upsilon}+\Upsilon \alpha) \sqrt{1+z^{\Upsilon}}-(\Upsilon+\alpha) z\right)^{\frac{\Upsilon(x-y)}{(x+\Upsilon+\alpha-y)}}}
$$

$t_{t r}=\left(c_{r}\left(\frac{\tau_{c}}{\gamma}\right)^{(x+r+\alpha)}\right)^{\frac{1}{(x+r+\alpha-y)}}$

$$
\frac{\left((\boldsymbol{r}+\boldsymbol{r} \alpha) \sqrt{1+z^{r}}-z(\boldsymbol{r}+\alpha)\right)^{\frac{(r+\alpha)}{(x+r+\alpha-y)}}}{\left(\boldsymbol{r}(1+\alpha) \sqrt{1+z^{r}}-z(1+\alpha)\right)^{\frac{(1+\alpha)}{(x+r+\alpha-y)}}}
$$

انديس tr، نشاندهندهى شرايط آستانهى حركت است. در روابط ذكرشده با

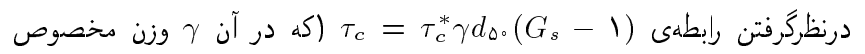

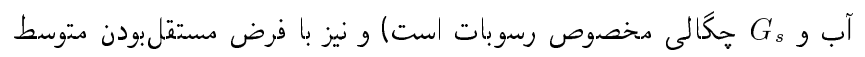

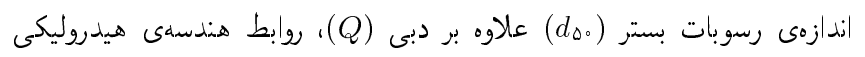

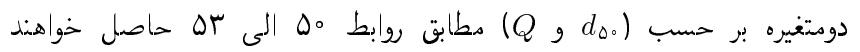

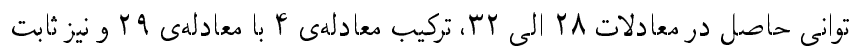

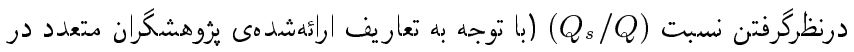

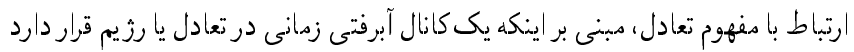
كه در آن نسبت

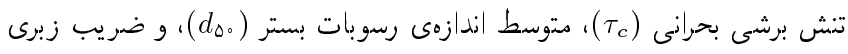

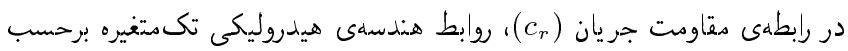

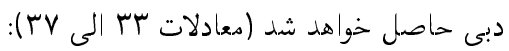

$\left(P_{b e d}\right)_{m}=e Q^{\left(\left(1-\beta_{\curlyvee} / \beta_{\curlyvee}\right) /(x+\Upsilon+\alpha-y)\right)+\left(\beta_{\curlyvee} / \beta_{\digamma}\right)}$

$D_{m}=c Q^{\left(\left(1+\frac{\left(1-\beta_{\Upsilon}\right)}{\beta_{r}}\right) /(x+r+\alpha-y)\right)+\frac{\left(\beta_{\Upsilon}-1\right)}{\beta_{\Upsilon}}}$

$S_{m}=t Q^{-\left(\left(1+\frac{\beta_{1}}{\beta_{\digamma}}\right) /(x+r+\alpha-y)\right)+\frac{\beta_{1}}{\beta_{\digamma}}}$

$V_{m}=k Q^{\left(\left(x+\alpha-y-\frac{\beta_{\digamma}}{\beta_{\digamma}}\right) /(x+r+\alpha-y)\right)+\frac{\beta_{\digamma}}{\beta_{\digamma}}}$

$W_{m}=a Q^{\left(\left(1-\frac{\beta_{\Delta}}{\beta_{\digamma}}\right) /(x+r+\alpha-y)\right)+\left(\beta_{\circlearrowright} / \beta_{\Upsilon}\right)}$

كه در روابط مذكور، ضرايب a، c، k، $t$ و e برابر هستند با:

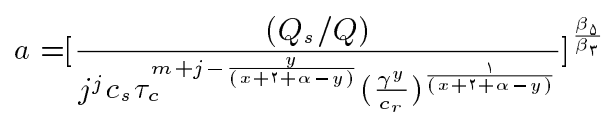
$\left(\frac{\gamma^{y}}{c_{r} \tau_{c}^{y}}\right)^{\frac{1}{(x+r+\alpha-y)}}$

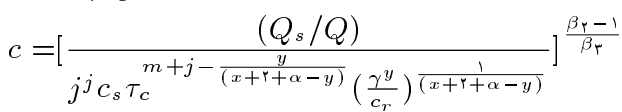

$\left(\frac{\gamma^{y}}{c_{r} \tau_{c}^{y}}\right)^{\frac{1}{(x+r+\alpha-y)}}$

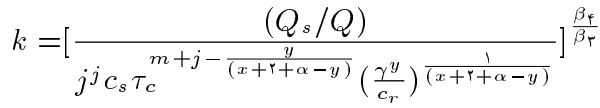
$\left(c_{r}^{r}\left(\frac{\tau_{c}}{\gamma}\right)^{r y}\right)^{\frac{1}{(x+r+\alpha-y)}}$

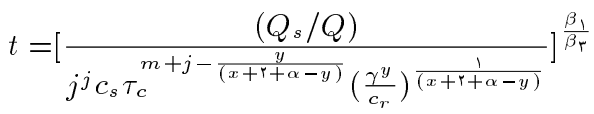
$\left(c_{r}\left(\frac{\tau_{c}}{\gamma}\right)^{x+\alpha+r}\right)^{\frac{1}{(x+r+\alpha-y)}}$

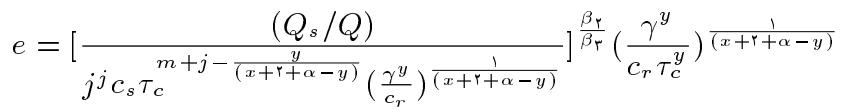
بهمنظور تعيين روابط هندسهى هيدروليكى دومتغيره بر حسب بارامترهاى دبى و

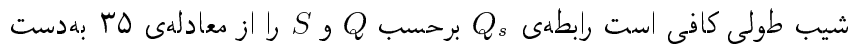

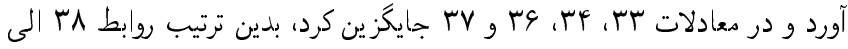
أ أ را خواهيم دراشت:

$\left(P_{b e d}\right)_{m}=e^{\prime} S^{\beta_{\curlyvee} / \beta_{\curlywedge}} Q^{\left(1+\beta_{\curlyvee} / \beta_{\curlywedge}\right) /(x+\Upsilon+\alpha-y)}$

$D_{m}=c^{\prime} S^{\frac{\beta_{r}-1}{\beta_{1}}} Q^{\left(1+\frac{\beta_{\gamma}-1}{\beta_{1}}\right) /(x+r+\alpha-y)}$

$V_{m}=k^{\prime} S^{\beta_{\digamma} / \beta_{\curlywedge}} Q^{\left(x+\alpha-y+\left(\beta_{\digamma} / \beta_{\uparrow}\right)\right) /(x+r+\alpha-y)}$

$W_{m}=a^{\prime} S^{\frac{\beta_{0}}{\beta_{1}}} Q^{\left(1+\frac{\beta_{0}}{\beta_{1}}\right) /(x+r+\alpha-y)}$

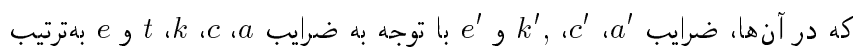

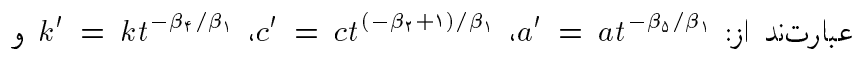
$. e^{\prime}=e t^{-\beta_{\Upsilon} / \beta_{1}}$ 
جدول r. مقايسهى روابط هندسهى هيدروليكى در حد هايينى

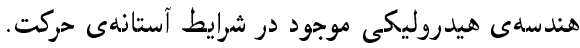

\begin{tabular}{|c|c|c|}
\hline روابط هندسهى هيدروليكى & نوع رابطه & 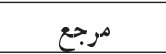 \\
\hline$W \propto Q^{\circ, 4 \uparrow \sim \circ / 0}$ & \multirow{4}{*}{ تحليلى ـ تكىتغيره } & \multirow{4}{*}{ مطالعهى حاضر } \\
\hline$D \propto Q^{\circ, 4+\sim 0, \Delta \circ}$ & & \\
\hline$S \propto Q^{-(0,00 \sim 0, \mu \varphi)}$ & & \\
\hline$V \propto Q^{\circ} \sim \circ, 1$ & & \\
\hline$W \propto d_{\Delta_{0}}^{-(0, r \Delta \sim 0, r r)} Q^{0, \mu \varphi \sim 0, \Delta}$ & \multirow{4}{*}{ تحليلى- دومتغيره } & \multirow{4}{*}{ مطالعهى حاضر } \\
\hline $\begin{array}{l}D \propto d_{\Delta_{0}}^{-(0, r \Delta \sim \circ, r r)} Q^{0, \mu \varphi \sim 0, \Delta \circ} \\
S \propto d^{1, r \sim 1, r \Delta} O_{-(0, \Delta \sim 0, \mu \varphi)}\end{array}$ & & \\
\hline$S \propto d_{\Delta_{0}} \quad Q^{-(0,0 \sim 0, \pi)}$ & & \\
\hline$V \propto d_{\Delta^{\circ}}^{0,4 \uparrow \sim 0, \Delta^{\circ}} Q^{\circ} \sim 0,11$ & & \\
\hline$W \propto Q^{\circ, 0}$ & \multirow{4}{*}{ نيمةتحليلى - تكىتغيره } & \multirow{4}{*}{ 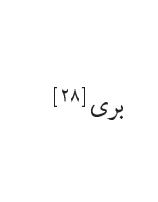 } \\
\hline$D \propto Q^{\circ, \uparrow A r}$ & & \\
\hline$S \propto Q^{-0, \text { FAr }}$ & & \\
\hline$V \propto Q^{\circ, 0 Y r}$ & & \\
\hline$W \propto d^{-0,10} Q^{0,49}$ & \multirow{4}{*}{ تحليلى - دومتغيره } & \multirow{4}{*}{ ل } \\
\hline$D \propto d^{-0,10} Q^{\circ, 49}$ & & \\
\hline$S \propto d^{1,10} Q^{-0,49}$ & & \\
\hline$V \propto d^{\circ, r} Q^{\circ, \wedge \wedge}$ & & \\
\hline
\end{tabular}

d قطر متوسط ذرات رسوبى بر حسب ميلىمتر است.

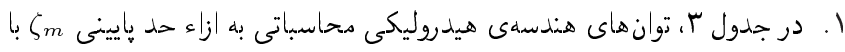

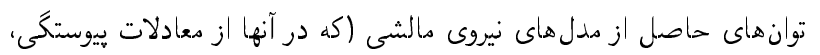

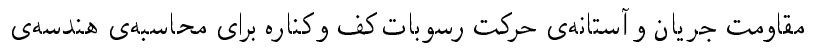

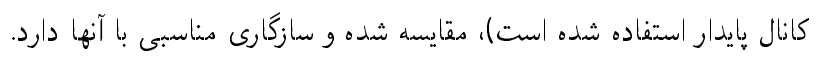

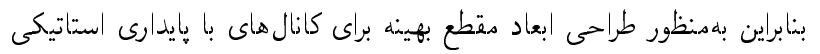

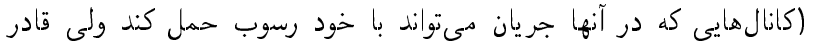

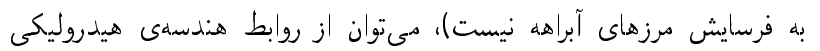

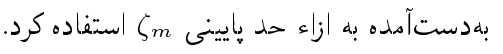

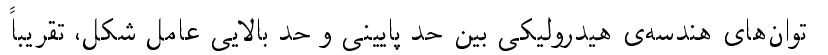

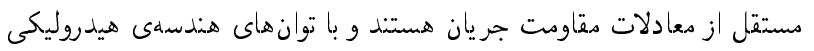

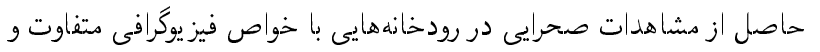

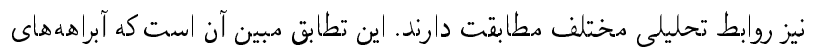

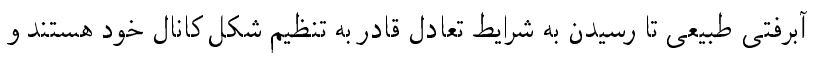

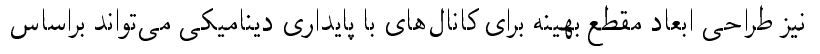
روابط مذكور صورت كيرد.

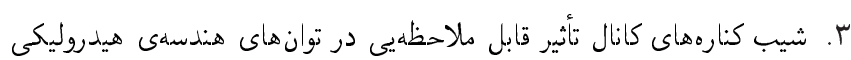

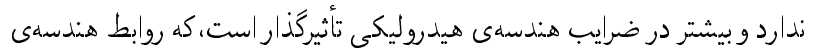

$$
\text { هيدروليكى تجربى موجود، [بَ] نيز مؤيد اين امر استى }
$$

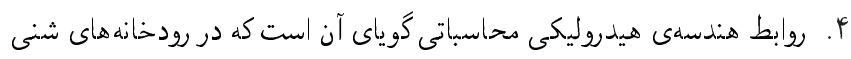

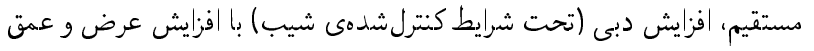

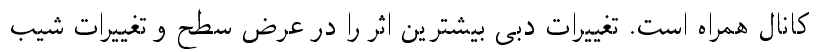
طولى، بيشترين اثر را در عمق كانال مى كذارند.
$S_{t r} \propto d_{\delta^{\circ}}^{(x+r+\alpha) /(x+r+\alpha-y)} Q^{-1 /(x+r+\alpha-y)}$

$D_{t r} \propto d_{0_{0}}^{-y /(x+r+\alpha-y)} Q^{1 /(x+r+\alpha-y)}$

$V_{t r} \propto d_{\delta^{\circ}}^{r y /(x+r+\alpha-y)} Q^{(x+\alpha-y) /(x+r+\alpha-y)}$

$W_{t r} \propto d_{\Delta^{\circ}}^{-y /(x+r+\alpha-y)} Q^{1 /(x+r+\alpha-y)}$

با توجه به دعادلات \&4 الى كسه، توانهاى هندسهى هيدروليكى تك متغيره در شرايط

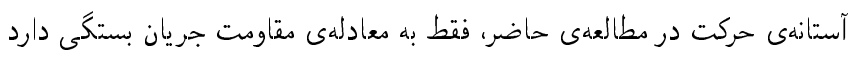

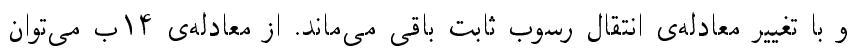

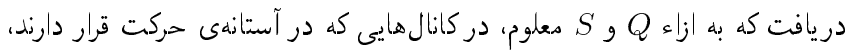

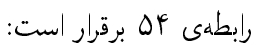

$\left(\tau_{\circ}\right)_{t r}=\max \tau_{\circ}(\zeta)=\tau_{c}$

هطابق sعادلهى Of بf به ازاء حد هإيينى

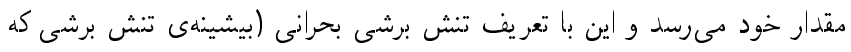

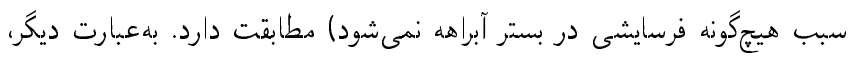

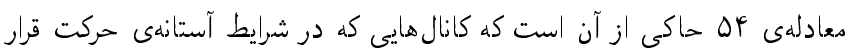

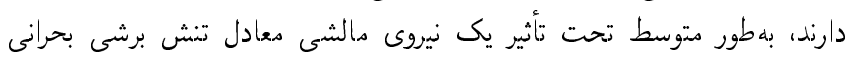

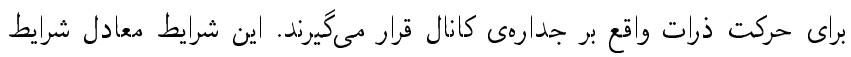

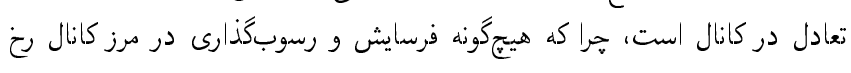

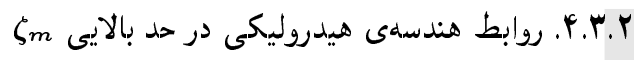

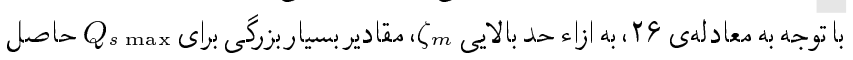

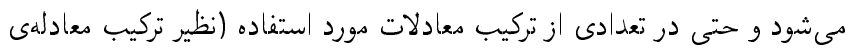

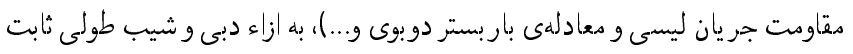
براى آبراهه،

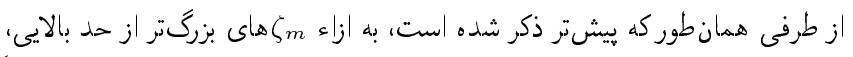

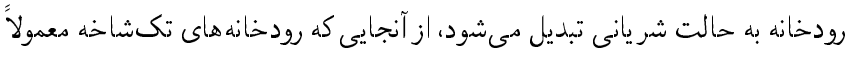

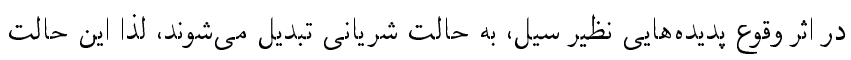

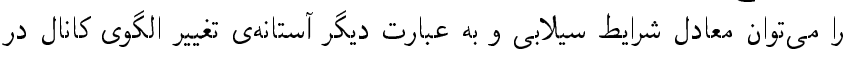

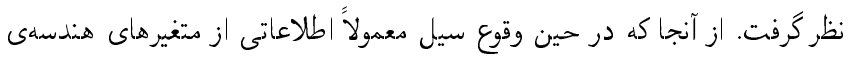

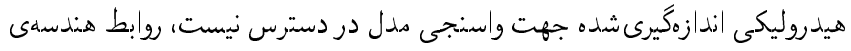

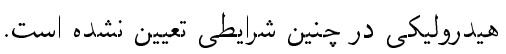

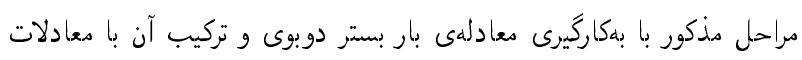

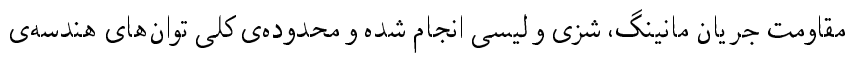
هيدروليكى حاصل از تركيب دعادلات مذكور به ازاء تغييرات زاويهى كنارهى كان كانال

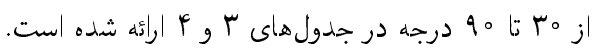

\section{F.r. F. مقايسهى روابط هندسهى هيدروليكى محاسباتى با روابط} موجود

در اين قسمت بهنظور بررسى صحت روابط هندسهى هيدروليكى حاصل از مدل

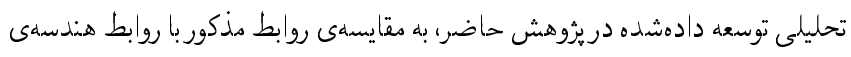
هيدروليكى تجربى و تحليلى موجود برداخته شده است. اهم نتايج بهدست آمده از از جدولهاى ب و با به اين شرح است: 


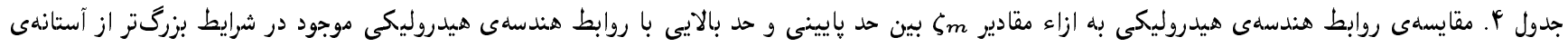

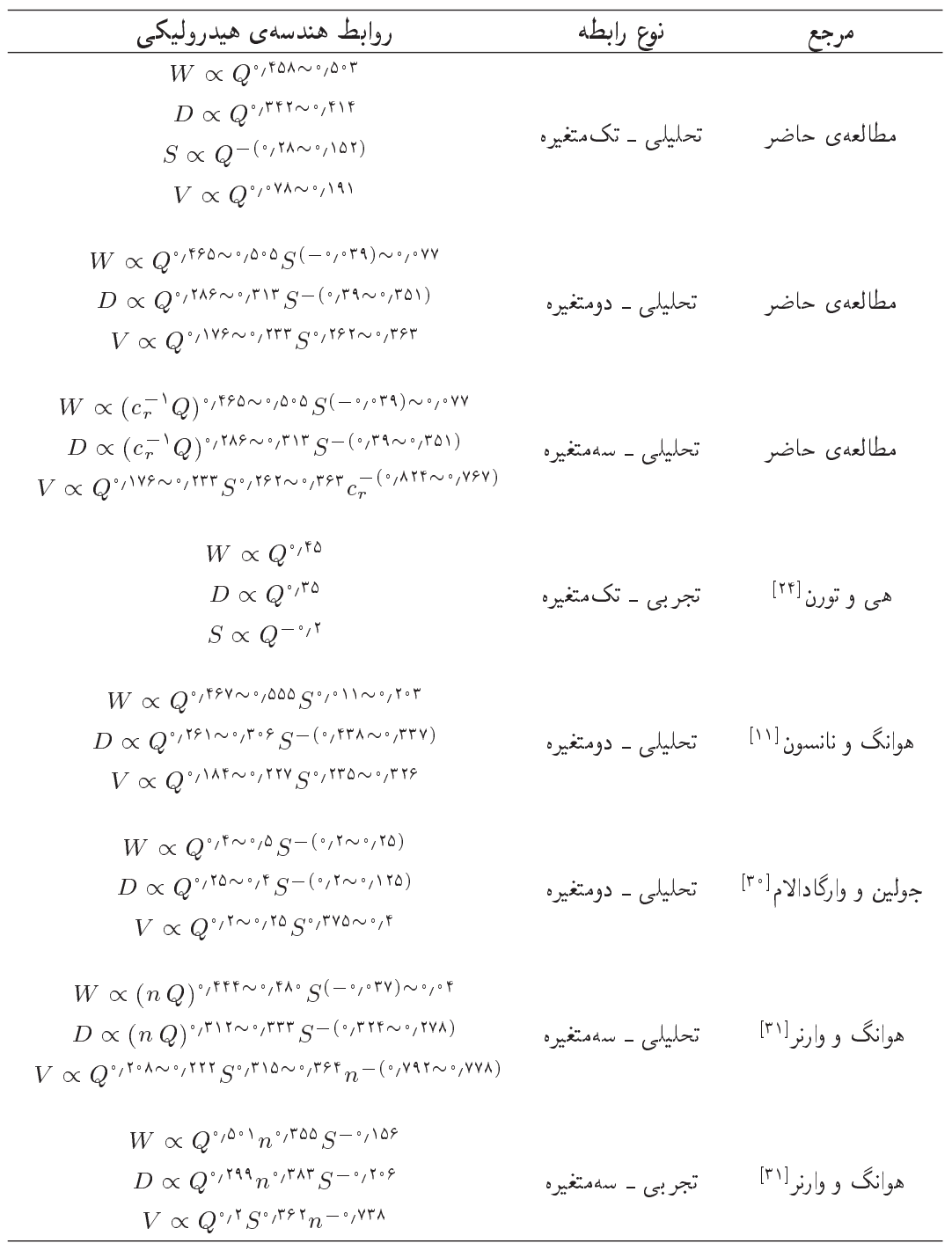

جدول ه. محدودهى دادهاى مورد استفاده (مرجع دادها: هى و تورن).[ץ+[

\begin{tabular}{|c|c|c|}
\hline مشخصات جريان & توضيحات & محدودهى مقادير مورد استفاده \\
\hline$Q_{b f}\left(\mathrm{~m}^{r} / \mathrm{s}\right)$ & مبى مقطع ير & $r r q-r, q$ \\
\hline$D_{\diamond}(\mathrm{m})$ & متوسط اندازهى مواد بستر & $0,1 \vee q-0,014$ \\
\hline$W(\mathrm{~m})$ & عرض مقطع ير & $v \vee / 10-0,0$ \\
\hline$d_{m}(\mathrm{~m})$ & بيشينهى عمق جريان & $\Delta, r \Delta-0, v v$ \\
\hline$S$ & شيب طولى كانال & $0,0 Y I F G V-0,00119$ \\
\hline$n$ & ضريب زبرى مانينى & $0,0 V-0,0 \mathrm{YF}$ \\
\hline
\end{tabular}

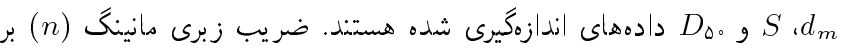

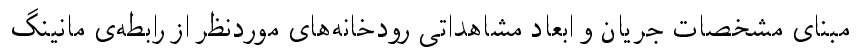

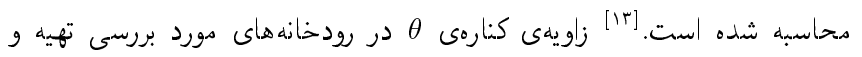

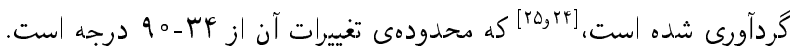

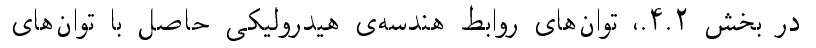

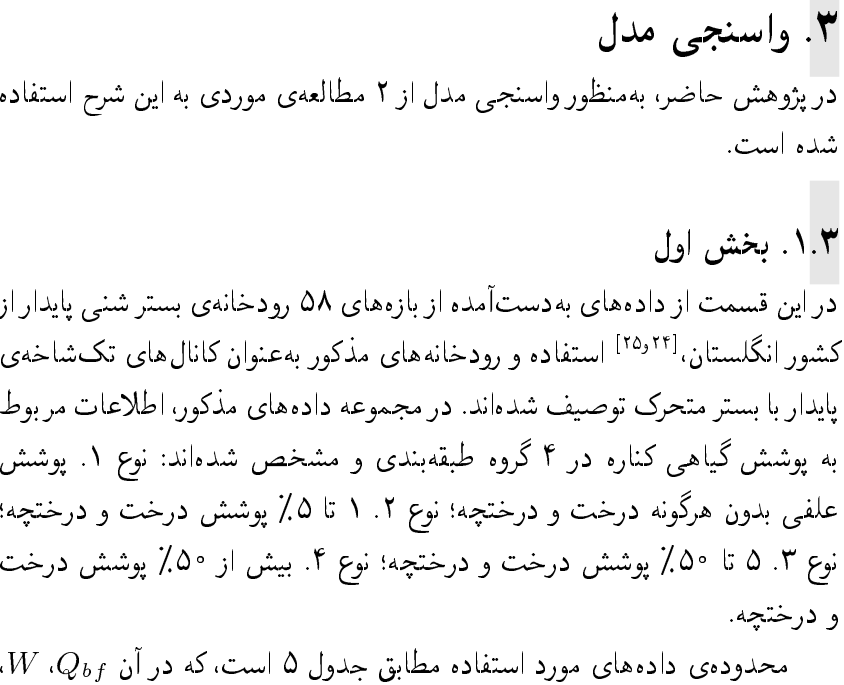

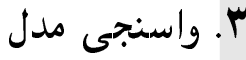
دحدودمى دادههاى مورد استفاده هطابق جدول ه است، كه در آن Waf 
مطابق جدول 9، على رغم تطابق مناسب توانهاى هندسهى هيدروليكى حاصل

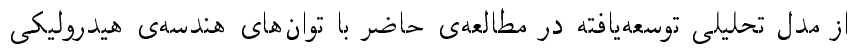

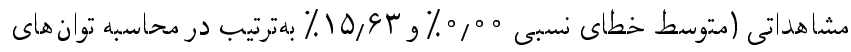

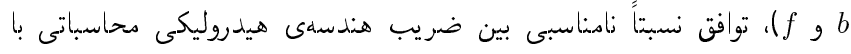

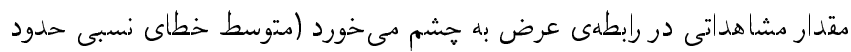

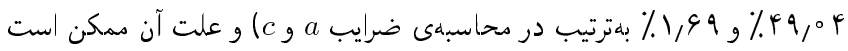

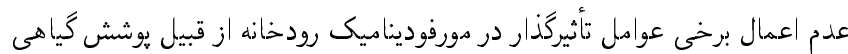
كناره در مدل بيشنهادى باشد. باشي.

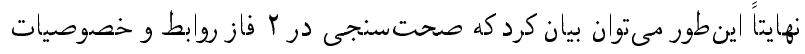

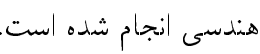

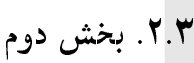

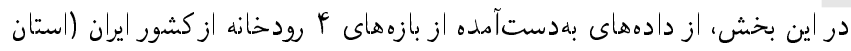

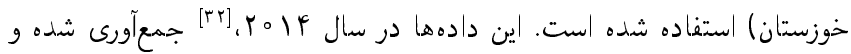

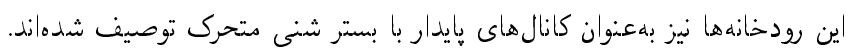

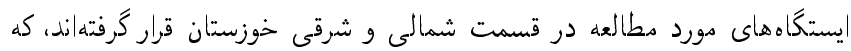

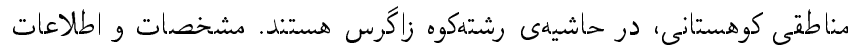

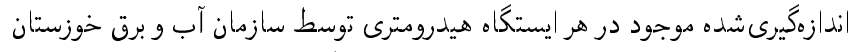

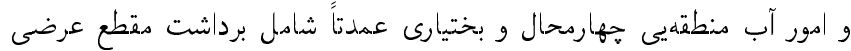

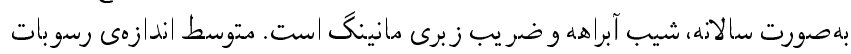

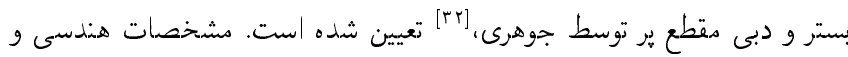

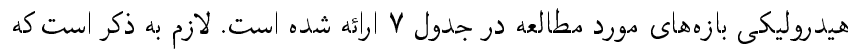

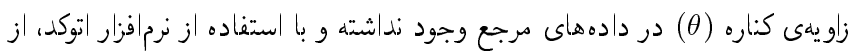

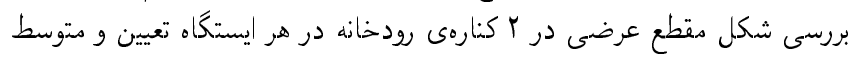

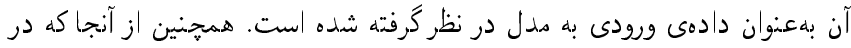

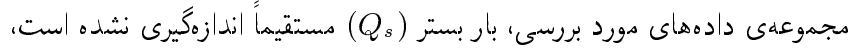

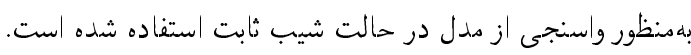

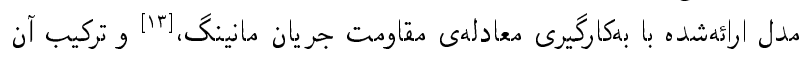

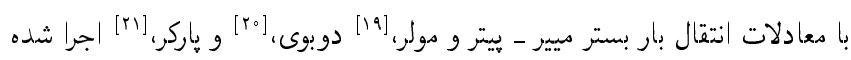

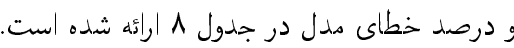

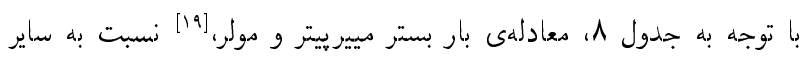

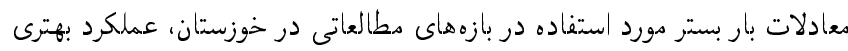

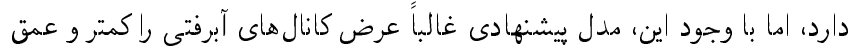
را بيشتر از مقادير مشاهداتى تخمين زده است.

\section{f أ. نتيجهكيرى}

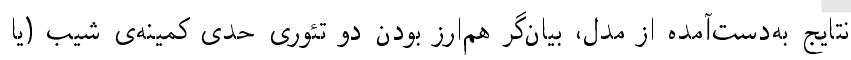

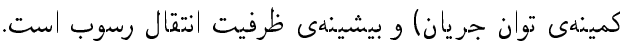

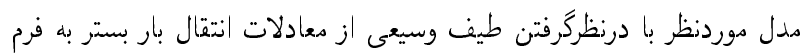

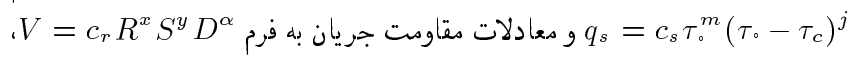

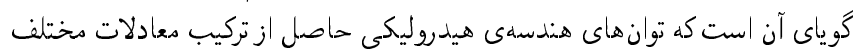

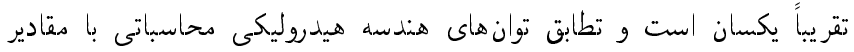

حاصل از مطالعات ساير بروهشكُران كه از دادهاي مشاهداتى حاصل شده است،

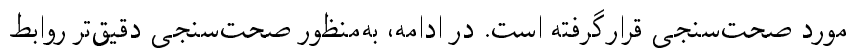

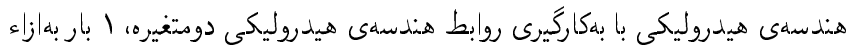

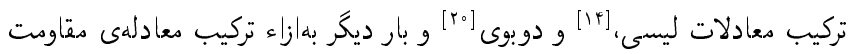

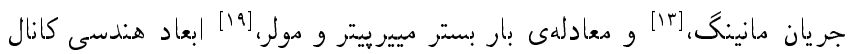

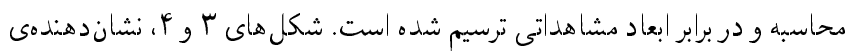

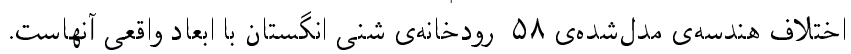

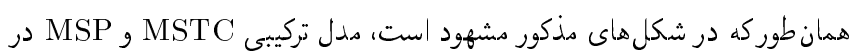

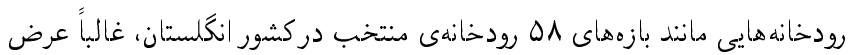

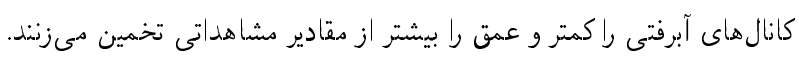

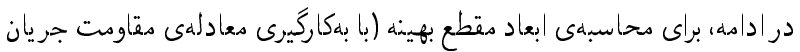

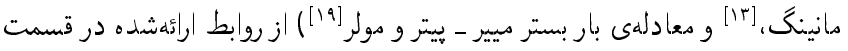

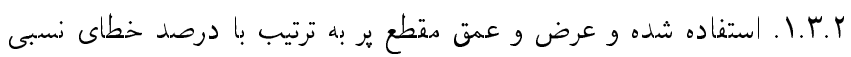

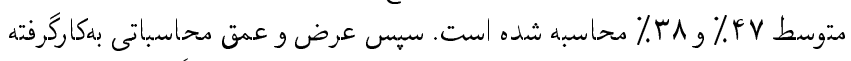

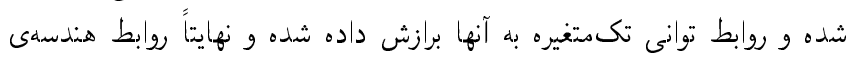

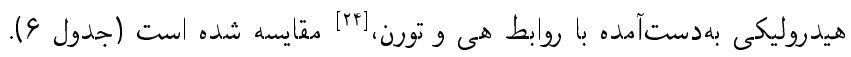

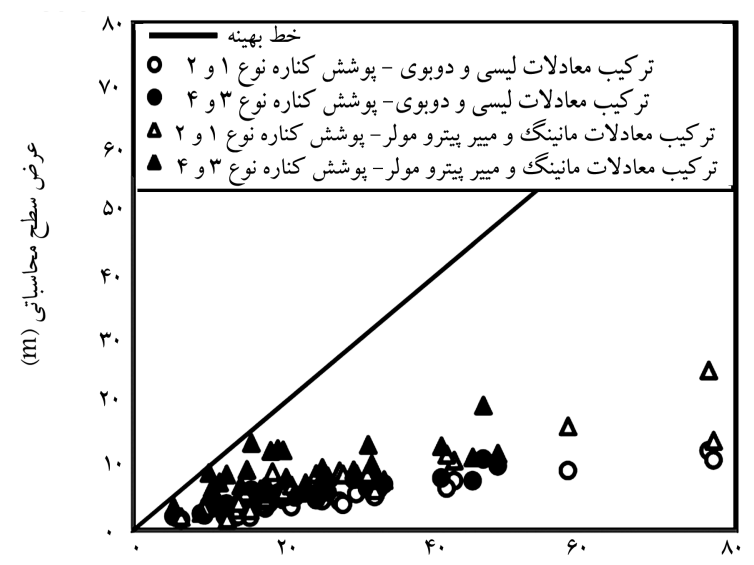

عرض سطح مشاهداتى (m)

شكل r. مقايسهى عرض مقطع ير محاسباتى و مشاهداتى.

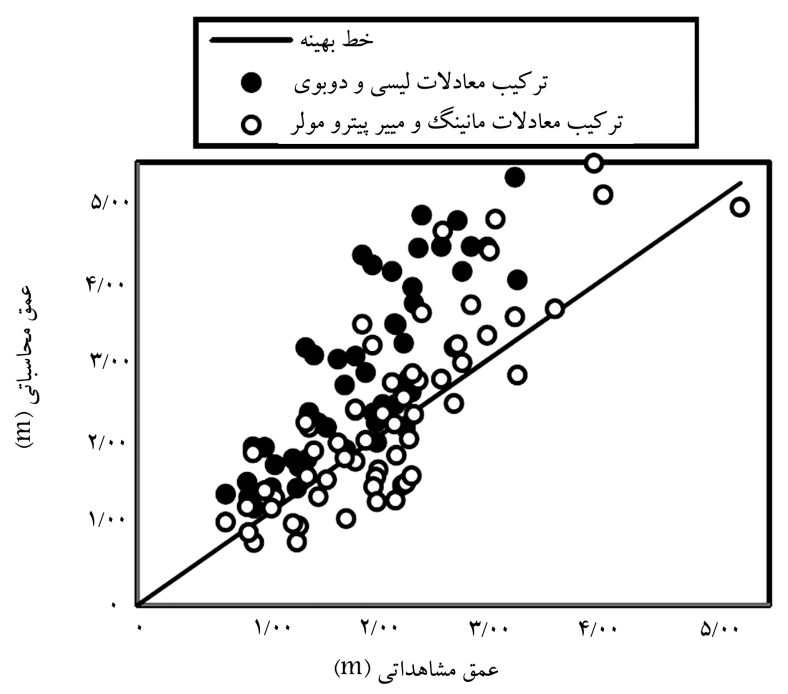

شكل fا. مقايسهى عمق مقطع ير محاسباتى و مشاهداتى. 
جدول 9. مقايسهى روابط توانى محاسباتى و مشاهداتى (سيستم SI).

\begin{tabular}{|c|c|c|c|c|c|c|}
\hline \multirow{2}{*}{$\begin{array}{c}\text { ضر يب تعيين } \\
\left(R^{r}\right)\end{array}$} & \multicolumn{4}{|c|}{$D=c Q^{f}, W=a Q^{b}$} & \multirow{2}{*}{ مشخصات كانال } & \multirow{2}{*}{ 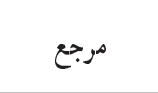 } \\
\hline & $f$ & $c$ & $b$ & $a$ & & \\
\hline $0, v \& q$ & - & - & 0,40 & 1,19 & عرض مقطع ير (W) & \\
\hline $0,9 \circ 0$ & $0, r v$ & $0,0 \Lambda$ & - & - & عمق ماكزيمم (D) & \\
\hline 0,101 & - & - & 0,190 & $r, 90$ & عرض مقطع ير (W) & \\
\hline $0, V \wedge \vee$ & $0, \mu r$ & 0,09 & - & - & عمق ماكزيمم (D) & \\
\hline
\end{tabular}

جدول V. مشخصات مقطع ير كانال رودخانه در † بازهى مطالعاتى در استان خوزستان (ايران).

\begin{tabular}{|c|c|c|c|c|c|c|c|c|}
\hline $\begin{array}{c}\text { عمق متوسط } \\
\text { (m) }\end{array}$ & عرض سطح & زاويه كناره & ضر يب زبرى & متوسط اندازه مواد & شولى & دبى مقطع ير & رودان & ايستگًاه \\
\hline$\Lambda, \mu \varphi$ & $G Y, \Delta F$ & rr & 0,041 & $0,0 \Delta r G$ & $0,000 \mathrm{~V}$ & $1 r r \circ, 91$ & كارون & سوسن \\
\hline$r, q V$ & $V Y, \Delta r$ & rr & $0,0 \mathrm{HF}$ & 0,0 YAT & 0,001149 & $r v r, \circ \Lambda$ & آب شيرين & خيرآباد \\
\hline$r, 99$ & $11 V, 9 \Lambda$ & $\Delta r$ & $0,0 M 1$ & $0,0 \mu \circ \Delta$ & $0,0 \circ \mu \circ r$ & $10 r q, 09$ & مارون & جمنظام \\
\hline 1,00 & $\mid r, \varphi$ & $\Delta F$ & 0,000 & 0,0499 & 0,0049 & $11, r V$ & زال & يّل زال \\
\hline
\end{tabular}

تغيير مىكند. در واقع در رودخانهاى عريض با كاهش اثر يوشش گياهى كناره در

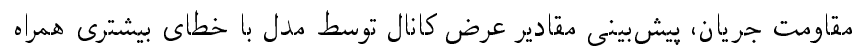

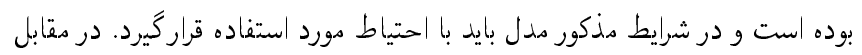

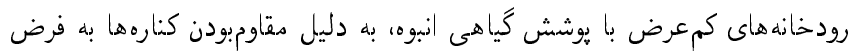

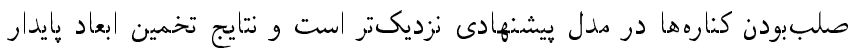
توسط ددل، در هنين رودخانههايى دقت بيشترى دارند.

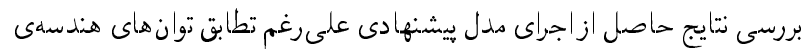

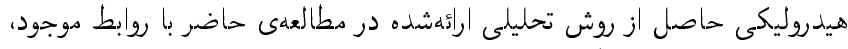

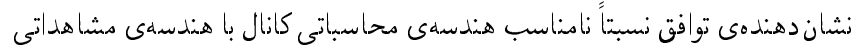

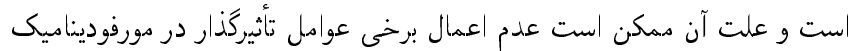
رودخانه از قبيل يوشش كياهى كناره باشد.

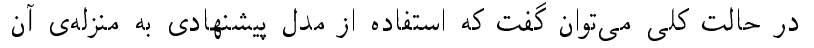

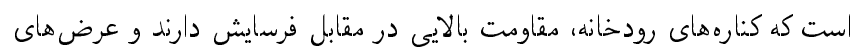

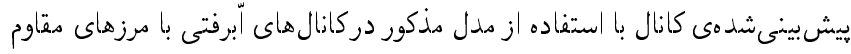

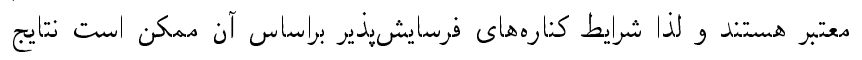

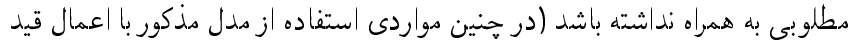

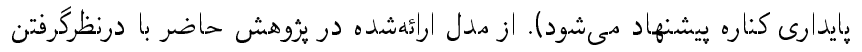

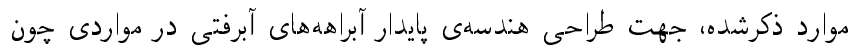

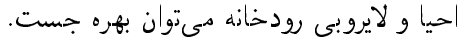

جدول ^. متوسط درصد خطاى نسبى محاسبات مدل.

\begin{tabular}{|c|c|c|}
\hline \multicolumn{2}{|c|}{ درصد خطاى نسبى متوسط } & معادلهى انتقال بار \\
\hline عمق متوسط & عرض مقطع ير & بستر \\
\hline$r r, 90$ & $(r \Delta, \Delta V)$ & ميير - ييتر و دولر [19] \\
\hline$r \Delta, \Delta \varphi$ & $(r T, \varphi \Delta)$ & 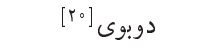 \\
\hline$r q, \Delta v$ & $(r q, r \vee)$ & 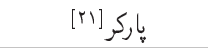 \\
\hline
\end{tabular}

مشاهداتى نشان مى دهد كه آبراهه هاى آبرفتى طبيعى تا رسيدن به شرايط تعادل،

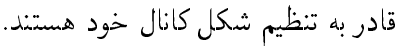

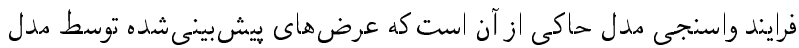

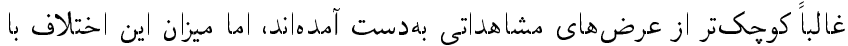

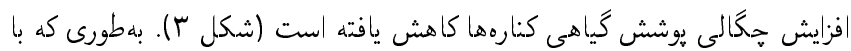

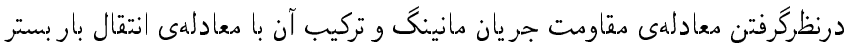

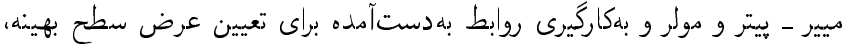

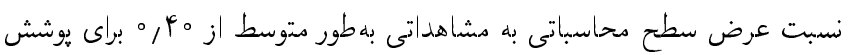

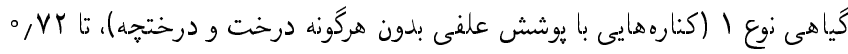

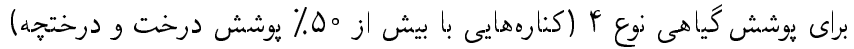

1. at-a-station hydraulic geometry

2. downstream hydraulic geometry
3. extrenal hypotheses

4. 4. 4. maximum sediment transport capacity

5. maximum flow efficiency

6. minimum stream power

7. minimum variance 
8. minimum energy dissipation rate

9. minimum unit stream power

10. riprap

\section{منابع (References)}

1. Millar, R.G. "Development of an analytical model of river response", MSc thesis, University of Queensland, Brisbane, Australia (1984).

2. Leopold, L.B. and Maddock, T., The Hydraulic Geometry of Stream Channels and Some Physiographic Implications, US Geological Survey, Professional Paper 252, Washington, D.C., 57 p. (1953).

3. Leopold, L.B. and Langbein, W.B., The Concept of Entropy in Landscape Evolution, U.S. Government Printing Office, Professional paper 500-A (1962).

4. Langbein, W.B. "Geometry of river channels", Journal of the Hydraulics Division, ASCE, 90(HY2), pp. 301-311 (1964).

5. Pickup, G. "Adjustment of stream channel shape to hydrologic regime", Journal of Hydrology, 30(4), pp. 365373 (1976).

6. Kirkby, M.J., Maximum Sediment Efficiency as a Criterion for Alluvial Channels, In River Channel Changes, Gregory, K.J. (ed.), Chichester: Wiley, pp. 429-442 (1977).

7. Yang, C.T. "Potential energy and stream morphology", Water Resources Research, 7(2), pp. 311-322 (1971a).

8. Yang, C.T. and Molinas, A. "Sediment transport and unit stream power function", Journal of the Hydra. Division, ASCE, 108, pp. 774-793 (1982).

9. Chang, H.H. "Minimum stream power and river channel patterns", Journal of Hydrology, 41(3-4), pp. 303-327 (1979b).

10. Song, C.S.S. and Yang, C.T. "Minimum stream power: Theory", J. Hydraul. Eng., ASCE, 106(9), pp. 14771488 (1980).

11. Huang, H.Q. and Nanson, G.C. "A stability criterion inherent in laws governing alluvial channel flow", Earth Surface Processes and Landforms, 27(9), pp. 929-944 (2002).

12. Knighton, A.D. "Fluvial forms and processes", Earth Surface Processes and Landforms, Edward Arnold: London, 24(4), pp. 378-379 (1998).

13. Manning, R. "On the flow of water in open channels and pipes", Transactions of the Institution of Civil Engineers of Ireland, 20, pp. 161-207 (1891).

14. Lacey, G. "Flow in alluvial channels with sandy mobile beds", Proceedings of the Institute of Civil Engineers, London, Discussion on paper No. 6274, 11(2), pp. 145164 (1958).

15. Chadwick, A.J. and Morfett, J.C., Hydraulics in Civil and Environmental Engineering, Champman \& Hall (1995).
16. Brownlie, W.R. "Flow depth in sand-bed channels", Journal of Hydraulic Engineering, ASCE, 109(7), pp. 959-990 (1983).

17. Einstein, H.A., The Bed-Load Function for Sediment Transportation in Open Channel Flows, U.S. Department of Agriculture, Soil Conservation Service, Technical Bulletin No. 1026 (1950).

18. Yang, C.T., Sediment Transport: Theory and Practice, McGraw-Hill (1996).

19. Meyer-Peter, E. and Muller, R. "Formulas for bed load transport", In Proceedings of the 3rd Meeting of IAHR Stockholm, pp. 39-46 (1948).

20. DuBoys, P. "Le Rhone et les rivieres a lit affouillable", Annales des Ponts et Chaussees, Series, 5(18), pp. 141195 (1879).

21. Parker, G. "Hydraulic geometry of active gravel rivers", Journal of the Hydraulics Division, ASCE, 105(9), pp. 1185-1201 (1979).

22. Eaton, B.C. and Church, M. "Channel stability in bed load dominated streams with nonerodible banks: Inferences from experiments in a sinuous flume", Journal of Geophysical Research - Earth Surface, 114(F1), pp. 1-17 (2009).

23. Engelund, F. and Skovgaard, O. "On the origin of meandering and braiding in alluvial streams", Journal of Fluid Mechanics, 57(02), pp. 289-302 (1973).

24. Hey, R.D. and Thorne, C.R. "Stable channels with mobile gravel beds", Journal of the Hydraulic Engineering,

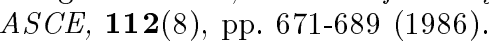

25. Darby, S.E. "Refined hydraulic geometry data for british gravel-bed rivers", Journal of Hydraulic Engineering, 131(1), pp. 60-64 (2005).

26. Yu, G. and Smart, G. "Aspect ratio to maximize sediment transport in rigid bank channels", Journal of Hydraulic Engineering, 129(12), pp. 927-935 (2003).

27. Simons, D.B. and Albertson, M.L. "Uniform water conveyance channels in alluvial materials", Journal of the Hydraulics Division, ASCE, 86(5), pp. 33-71 (1960).

28. Bray, D.I., Regime Equations for Gravel-Bed Rivers, In Hey, R. D.; Bathurst, J. C. and Thorne. C. R., (Editors), Gravel-bed Rivers: Fluvial Processes, Engineering and Management, John Wiley and Sons, Chichester, pp. 517552 (1982b).

29. Lane, E.W. "The design of stable channels", Trans., $A S C E, \mathbf{1 2 0}(2776)$, pp. 1234-1279 (1955b).

30. Julien, P.Y. and Wargadalam, J. "Alluvial channel geometry: Theory and applications", Journal of Hydraulic Engineering, ASCE, 121(4), pp. 312-325 (1995).

31. Huang, H.Q. and Warner, R.F. "The multivariate controls of hydraulic geometry; a causal investigation in terms of boundary shear distribution", Earth Surfaces Processes and Landforms, 20(2), pp. 115-130 (1995).

32. Johari, R. "Field observation of spatial variation degree of armoring in gravel bed-river and its impact on the hydraulic parameters", M. Sc. Thesis, Shahid Beheshti University, Tehran, Iran (in Persian) (2014). 